MKPH-T-00-17, ECT*-00-009

\title{
Generalized polarizabilities of the proton in a constituent quark model revisited
}

\author{
B. Pasquini, ${ }^{1}$ S. Scherer ${ }^{2}$ and D. Drechsel ${ }^{2}$ \\ ${ }^{1}$ ECT* ${ }^{*}$ European Centre for Theoretical Studies in Nuclear Physics and Related Areas, \\ Strada delle Tabarelle 286, I-38050 Villazzano (Trento), Italy \\ ${ }^{2}$ Institut für Kernphysik, Johannes Gutenberg-Universität, J. J. Becher-Weg 45, D-55099 \\ Mainz, Germany
}

(August 22, 2000)

\begin{abstract}
We study low-energy virtual Compton scattering off the proton within the framework of a nonrelativistic constituent quark model. The Compton tensor is divided into two separately gauge-invariant contributions. The first consists of the groundstate propagation in the direct and crossed channels together with an appropriately chosen term to satisfy gauge invariance. The residual part contains the relevant structure information characterized by the so-called generalized polarizabilities. We discuss two different schemes to obtain the generalized polarizabilities from the residual term. Explicit predictions for the generalized polarizabilities are presented for the IsgurKarl model. Our results are compared with previous predictions in that model as well as other approaches.
\end{abstract}

12.39.Jh,13.60.Fz,14.20.Dh 


\section{INTRODUCTION}

The electromagnetic polarizabilities $\bar{\alpha}$ (electric) and $\bar{\beta}$ (magnetic) of real Compton scattering (RCS) [1] describe, to leading order in the photon frequency, the modeldependent response of a spin-0 or spin- $1 / 2$ system beyond the low-energy theorem (LET) 24. Within a classical framework these quantities are accessible to an intuitive interpretation as a "measure of the stiffness or rigidity of a system [5]." There have been considerable experimental efforts to determine the proton polarizabilities from Compton scattering off the proton [6 9]. Until recently, the most precise values for the proton polarizabilities were derived in the work of MacGibbon et al. [9] and analyzed by means of dispersion relations at fixed $t$ [10]. The results were $\bar{\alpha}_{p}=(12.1 \pm 0.8 \pm 0.5) \times 10^{-4} \mathrm{fm}^{3}$ and $\bar{\beta}_{p}=(2.1 \mp 0.8 \mp 0.5) \times 10^{-4} \mathrm{fm}^{3}$. The analysis of Ref. [9] made use of the Baldin sum rule [11] which relates the sum of the two polarizabilities to the total photoabsorption cross section. The sum rule constraint was $\bar{\alpha}_{p}+\bar{\beta}_{p}=(14.2 \pm 0.5) \times 10^{-4} \mathrm{fm}^{3}$ [12 , while more recent analyses found $(13.69 \pm 0.14) \times 10^{-4} \mathrm{fm}^{3}[13]$ and $[14.0 \pm(0.3-0.5)] \times 10^{-4} \mathrm{fm}^{3}$ [14]. New real-Compton-scattering data below pion-production threshold have been measured at the Mainz Microtron (MAMI). The new global fit including these data results in the values $\bar{\alpha}_{p}=(12.24 \pm 0.24 \pm 0.54 \pm 0.37) \times 10^{-4} \mathrm{fm}^{3}$ and $\bar{\beta}_{p}=(1.57 \pm 0.25 \pm 0.52 \pm 0.37) \times 10^{-4}$ $\mathrm{fm}^{3}$ [15. As there is no free neutron target the experimental information on the neutron polarizabilities is much less certain. Results for the electric polarizability have been obtained from low-energy neutron scattering off the Coulomb field of a heavy nucleus [16 19]. Alternatively, the quasifree Compton scattering reaction $d\left(\gamma, \gamma^{\prime} n\right) p$ [20 22] as well as elastic deuteron Compton scattering [23] have been investigated to extract information on the neutron polarizabilities.

Clearly, the concept of polarizabilities is open to generalizations in, at least, two directions. One possibility consists of investigating higher-order terms in the expansion of the RCS amplitude. For example, for a spin-1/2 system one finds four additional constants (spin polarizabilities) at third order 24 27] and four terms for the spin-averaged amplitude at fourth order [27,28]. Another option is to allow at least one photon to be virtual. This second generalization, which was already discussed in the 1970's in the context of nuclei [29], has recently been applied to the nucleon [30,31].

As in all studies with electromagnetic probes, the inclusion of virtual photons substantially increases the possibilities to investigate the structure of the target. The use of a virtual photon allows one to access longitudinal degrees of freedom and to vary the threemomentum and energy transfer to the target independently. The potential of investigating electron-proton bremsstrahlung as a source of the virtual Compton effect amplitude was already noticed by Berg and Lindner [32] in the 1960's. In its most general form for one real and one virtual photon, this amplitude can be described by twelve form factors of three invariants [32]. A generalized low-energy theorem (GLET) analogous to the LET of RCS [2 [4] was derived in Ref. [33], where it was shown that, up to and including second order in the momenta, all twelve amplitudes for the proton can be expressed in terms of the proton mass $M$, the anomalous magnetic moment $\kappa$, the electromagnetic Sachs form factors $G_{E}$ and $G_{M}$, the mean square electric radius $r_{E}^{2}$, and the RCS electromagnetic polarizabilities $\bar{\alpha}$ and $\bar{\beta}$.

In Ref. [30, the model-dependent response beyond the LET was analyzed by means 
of a multipole expansion. Only terms contributing to first order in the frequency of the outgoing real photon were kept, and the result was expressed in terms of ten generalized polarizabilities (GPs) which are functions of the three-momentum of the virtual photon in the initial state. Further progress has been made with respect to implementing the constraints due to the discrete symmetries in combination with particle crossing. In Refs. [34 36] it was shown that only six of the originally ten GPs are independent if chargeconjugation symmetry is combined with particle crossing.

Following the very first calculation in the framework of a nonrelativistic quark model 30,37, there have been numerous predictions for the GPs within various approaches, including phenomenological Lagrangians [38,39], the linear sigma model [40], chiral perturbation theory [41], the Skyrme model [42], a relativistic constituent quark model [43], and the so-called small scale expansion 44. On the experimental side, first evidence for virtual Compton scattering events was reported in Ref. 45. The first results for two structure functions involving linear combinations of GPs at $Q^{2}=0.33 \mathrm{GeV}^{2}$ have been obtained from a dedicated VCS experiment at MAMI [46]. Further experiments probing the GPs at different values of momentum transfer are underway at Jefferson Lab [47] and MIT-Bates 48.

Our work is organized as follows. We start out in Sec. II with a general discussion of the (virtual) Compton scattering tensor for a nonrelativistic system of $N$ particles. We propose a separation into two individually gauge-invariant pieces. The first part consists of the groundstate propagating in the direct and crossed channels, supplemented by an appropriately chosen term to satisfy gauge invariance. The residual part contains the structure information contributing to the GPs. Section III deals with the multipole expansion of the Compton tensor and the definition of the GPs according to Ref. [30]. Two schemes are presented to identify those pieces of the residual term that actually yield contributions to the GPs. In Sec. IV we reconsider the calculation of the GPs in the framework of the Isgur-Karl model, and Sec. V contains a short summary and some conclusions. The more technical details can be found in the appendices.

\section{HADRONIC COMPTON SCATTERING TENSOR}

In this section we discuss the formalism to describe both real and virtual Compton scattering $\gamma^{*}(\omega, \vec{q})+N\left(E_{i}, \vec{p}_{i}\right) \rightarrow \gamma^{*}\left(\omega^{\prime}, \vec{q}^{\prime}\right)+N\left(E_{f}, \vec{p}_{f}\right)$ off a composite system, denoted by $N$, within the framework of nonrelativistic quantum mechanics. We only consider the main steps to derive the hadronic tensor of Compton scattering and refer the interested reader to Refs. [49 53] and the more recent work of Refs. [54,55] for a detailed discussion.

The starting point is the nonrelativistic Hamiltonian for a composite system of $N$ particles with masses $m_{\alpha}$,

$$
H_{0}=-\sum_{\alpha=1}^{N} \frac{\left(\vec{\nabla}_{\alpha}\right)^{2}}{2 m_{\alpha}}+\sum_{\alpha<\beta} V_{\alpha \beta},
$$

where $\vec{r}_{\alpha}$ and $-i \vec{\nabla}_{\alpha}$ refer, respectively, to the position and conjugate momentum of the particle $\alpha$ in the coordinate representation. We use natural units, i.e., $\hbar=c=1, e>0$, 
and $\alpha=e^{2} / 4 \pi \approx 1 / 137$. For simplicity, we only consider a local potential $V_{\alpha \beta}$, i.e., the interaction between the constituents does not contain momentum-dependent and/or exchange forces, and thus avoid the problem of exchange currents. The eigenstates of Eq. (四), denoted by $\left|X \vec{p}_{X}\right\rangle$, are normalized according to

$$
\left\langle X^{\prime} \vec{p}_{X^{\prime}} \mid X \vec{p}_{X}\right\rangle=\delta^{3}\left(\vec{p}_{X^{\prime}}-\vec{p}_{X}\right) \delta_{X^{\prime} X}
$$

where $X$ and $\vec{p}_{X}$ refer to internal quantum numbers and the total momentum of the system, respectively.

The interaction with an external electromagnetic field is introduced via minimal substitution. In addition, we include a coupling to an intrinsic magnetic dipole moment $\vec{\mu}_{\alpha}=e_{\alpha} \vec{\sigma}_{\alpha} / 2 m_{\alpha}$ of the constituents, where $\vec{\sigma}_{\alpha} / 2$ is the Pauli spin operator. The resulting electromagnetic interaction Hamiltonian in the Schrödinger representation reads

$$
\begin{aligned}
H_{I} & =H_{I, 1}+H_{I, 2}, \\
H_{I, 1} & =\int d^{3} x J^{\mu}(\vec{x}) A_{\mu}(\vec{x}), \\
H_{I, 2} & =\frac{1}{2} \int d^{3} x \int d^{3} x^{\prime} B^{\mu \nu}\left(\vec{x}, \vec{x}^{\prime}\right) A_{\mu}(\vec{x}) A_{\nu}\left(\vec{x}^{\prime}\right),
\end{aligned}
$$

where $A^{\mu}(\vec{x})$ is the second-quantized photon field and

$$
\begin{aligned}
& \vec{J}(\vec{x})=\sum_{\alpha=1}^{N} \frac{e_{\alpha}}{2 m_{\alpha}}\left[\delta^{3}\left(\vec{x}-\vec{r}_{\alpha}\right)\left(\frac{\vec{\nabla}_{\alpha}}{i}-\vec{\sigma}_{\alpha} \times \vec{\nabla}_{\alpha}\right)-\left(\frac{\overleftarrow{\nabla} \alpha}{i}+\vec{\sigma}_{\alpha} \times \overleftarrow{\nabla}_{\alpha}\right) \delta^{3}\left(\vec{x}-\vec{r}_{\alpha}\right)\right] \\
& \rho(\vec{x})=\sum_{\alpha=1}^{N} e_{\alpha} \delta^{3}\left(\vec{x}-\vec{r}_{\alpha}\right), \\
& B^{\mu 0}=B^{0 \nu}=0, \quad B^{i j}\left(\vec{x}, \vec{x}^{\prime}\right)=\delta_{i j} \sum_{\alpha=1}^{N} \frac{e_{\alpha}^{2}}{m_{\alpha}} \delta^{3}\left(\vec{x}-\vec{r}_{\alpha}\right) \delta^{3}\left(\vec{x}^{\prime}-\vec{r}_{\alpha}\right) .
\end{aligned}
$$

The total Hamiltonian $H=H_{0}+H_{\text {rad }}+H_{I}$, where $H_{\text {rad }}$ refers to the Hamiltonian of the radiation field, is time independent.

The hadronic Compton tensor is obtained by calculating the contributions of $H_{I, 2}$ and $H_{I, 1}$ in first-order and second-order perturbation theory, respectively,?

$$
M_{f i}^{\mu \nu}\left(q^{\prime}, q, \vec{p}\right)=S_{f i}^{\mu \nu}\left(q^{\prime}, q\right)+T_{f i}^{\mu \nu}\left(q^{\prime}, q, \vec{p}\right)
$$

where $q$ and $q^{\prime}$ refer to the four-momenta of the initial and final photons. In Eq. (9) we have also kept the dependence on a third independent three-momentum, namely, the

\footnotetext{
${ }^{1}$ We adopt a covariant notation, even though a calculation within the framework of nonrelativistic quantum mechanics does not provide a covariant result.

${ }^{2}$ For notational convenience we keep the four-momenta $q$ and $q^{\prime}$ as arguments, although their time components are related by energy conservation.
} 
average of the initial and final target momenta $\vec{p}=\left(\vec{p}_{i}+\vec{p}_{f}\right) / 2$. Usually this dependence is reexpressed in terms of the photon three-momenta when specifying the reference frame through $\vec{p}=\left\{\left(\vec{q}-\vec{q}^{\prime}\right) / 2, \overrightarrow{0},-\left(\vec{q}+\vec{q}^{\prime}\right) / 2\right\}$ for the laboratory, Breit, and center-of-mass frame, respectively. This issue is of importance in the context of photon crossing as will be shown later.

Our normalization convention for the Compton tensor follows from the S-matrix element of RCS, $\gamma(q, \lambda)+N\left(p_{i}, \sigma\right) \rightarrow \gamma\left(q^{\prime}, \lambda^{\prime}\right)+N\left(p_{f}, \sigma^{\prime}\right)$ :

$S_{f i}=\delta^{3}\left(\vec{p}_{f}-\vec{p}_{i}\right) \delta^{3}\left(\vec{q}-\vec{q}^{\prime}\right) \delta_{\sigma^{\prime} \sigma} \delta_{\lambda^{\prime} \lambda}-\frac{i}{8 \pi^{2} \sqrt{\omega \omega^{\prime}}} \delta^{4}\left(p_{f}+q^{\prime}-p_{i}-q\right) \epsilon_{\mu}^{\prime *}\left(\lambda^{\prime}\right) \epsilon_{\nu}(\lambda) M_{f i}^{\mu \nu}\left(q^{\prime}, q, \vec{p}\right)$.

Using the interaction representation with respect to $H_{0}+H_{\text {rad }}$, [? the first-order contribution is a sum of the "seagull" terms,

$$
S_{f i}^{\mu \nu}\left(q^{\prime}, q\right)=(2 \pi)^{3}\left\langle f \vec{p}_{f}\left|B^{\mu \nu}\left(q^{\prime}, q\right)\right| i \vec{p}_{i}\right\rangle
$$

where

$$
B^{\mu \nu}\left(q^{\prime}, q\right)=\frac{1}{2} \int d^{3} z\left[e^{-i \vec{q}^{\prime} \cdot \vec{z}} B^{\mu \nu}(\vec{z}, 0)+e^{i \vec{q} \cdot \vec{z}} B^{\nu \mu}(\vec{z}, 0)\right]
$$

The latter equation follows from translational invariance,

$$
B^{\mu \nu}\left(\vec{x}, \vec{x}^{\prime}\right)=e^{-i \vec{P} \cdot \vec{x}^{\prime}} B^{\mu \nu}\left(\vec{x}-\vec{x}^{\prime}, 0\right) e^{+i \vec{P} \cdot \vec{x}^{\prime}}
$$

where $\vec{P}$ refers to the total momentum operator of the composite system.

The second-order result from the direct and crossed channels reads

$$
\begin{aligned}
T_{f i}^{\mu \nu}\left(q^{\prime}, q, \vec{p}\right)= & (2 \pi)^{3} \sum_{X} \int d^{3} p_{X}\left\langle f \vec{p}_{f}\left|J^{\mu}(0)\right| X \vec{p}_{X}\right\rangle \frac{(2 \pi)^{3} \delta^{3}\left(\vec{p}_{X}-\vec{p}_{i}-\vec{q}\right)}{E_{f}\left(\vec{p}_{f}\right)+\omega^{\prime}-E_{X}\left(\vec{p}_{X}\right)}\left\langle X \vec{p}_{X}\left|J^{\nu}(0)\right| i \vec{p}_{i}\right\rangle \\
& +(2 \pi)^{3} \sum_{X} \int d^{3} p_{X}\left\langle f \vec{p}_{f}\left|J^{\nu}(0)\right| X \vec{p}_{X}\right\rangle \frac{(2 \pi)^{3} \delta^{3}\left(\vec{p}_{X}-\vec{p}_{i}+\vec{q}^{\prime}\right)}{E_{f}\left(\vec{p}_{f}\right)-\omega-E_{X}\left(\vec{p}_{X}\right)}\left\langle X \vec{p}_{X}\left|J^{\mu}(0)\right| i \vec{p}_{i}\right\rangle,
\end{aligned}
$$

with the energy of the intermediate state $\left|X \vec{p}_{X}\right\rangle$ given by $E_{X}\left(\vec{p}_{X}\right)=\vec{p}_{X}^{2} / 2 M+\Delta E_{X}, M$ being the target mass and $\Delta E_{X}$ the excitation energy of the corresponding state. For further reference we note that Eqs. (11) and (13) are symmetrical under photon crossing, $q^{\mu} \leftrightarrow-q^{\prime \mu}$ and $\nu \leftrightarrow \mu$. .

\footnotetext{
${ }^{3}$ Alternatively, we could have treated the electromagnetic field as a time-dependent external field and used the interaction representation with respect to $H_{0}$. Both approaches yield the same result.

${ }^{4}$ Sometimes the symmetry property $B^{\mu \nu}\left(\vec{x}, \vec{x}^{\prime}\right)=B^{\nu \mu}\left(\vec{x}^{\prime}, \vec{x}\right)$ is used to express Eq. (12) as $\int d^{3} z e^{-i \vec{q}^{\prime} \cdot \vec{z}} B^{\mu \nu}(\vec{z}, 0)$ which, however, is no longer manifestly symmetrical under photon crossing.
} 
By use of the standard procedure to separate center-of-mass and internal motions [51.53, the matrix element of the current operator may be written as

$$
\left\langle f \vec{p}_{f}\left|J_{\mu}(0)\right| i \vec{p}_{i}\right\rangle \equiv \frac{1}{(2 \pi)^{3}}\left\langle f\left|J_{\mu}(\vec{q}, \vec{p})\right| i\right\rangle
$$

where

$$
\begin{aligned}
\vec{J}(\vec{q}, \vec{p}) & =\vec{j}^{i n}(\vec{q})+\frac{\vec{p}}{M} \rho(\vec{q}), \\
J_{0}(\vec{q}, \vec{p}) & =\rho(\vec{q}),
\end{aligned}
$$

with $\vec{q}=\vec{p}_{f}-\vec{p}_{i}$. In Eq. (15), the intrinsic current operator $\vec{j}^{i n}(\vec{q})$ and the charge density operator $\rho(\vec{q})$ are

$$
\begin{aligned}
\vec{j}^{i n}(\vec{q}) & =\sum_{\alpha} \frac{e_{\alpha}}{2 m_{\alpha}}\left(\left\{e^{i \vec{q} \cdot \vec{r}_{\alpha}^{\prime}}, \vec{p}_{\alpha}^{\prime}\right\}+i \vec{\sigma}_{\alpha} \times \vec{q} e^{i \vec{q} \cdot \vec{r}_{\alpha}^{\prime}}\right), \\
\rho(\vec{q}) & =\sum_{\alpha} e_{\alpha} e^{i \vec{q} \cdot \vec{r}_{\alpha}^{\prime}}
\end{aligned}
$$

where $\{a, b\} \equiv a b+b a$ is the standard anticommutator, and $\vec{r}_{\alpha}^{\prime}$ and $\vec{p}_{\alpha}^{\prime}$ are the intrinsic coordinates and momenta of the particles relative to the center of mass,

$$
\begin{aligned}
& \vec{r}_{\alpha}^{\prime}=\vec{r}_{\alpha}-\vec{R}, \quad \vec{R}=\frac{1}{M} \sum_{\alpha} m_{\alpha} \vec{r}_{\alpha}, \\
& \vec{p}_{\alpha}^{\prime}=\vec{p}_{\alpha}-\frac{m_{\alpha}}{M} \vec{P}, \quad \vec{P}=\sum_{\alpha} \vec{p}_{\alpha} .
\end{aligned}
$$

We note that the intrinsic coordinates and momenta do not satisfy the canonical commutation relations,

$$
\left[r_{\alpha, i}^{\prime}, p_{\beta, j}^{\prime}\right]=i \delta_{i j}\left(\delta_{\alpha \beta}-\frac{m_{\beta}}{M}\right)
$$

From now on we assume that both initial and final states, denoted by $|0\rangle$ and $\left\langle 0^{\prime}\right|$, respectively, correspond to the ground state of the system. However, we explicitly allow for a change in the spin projection. Inserting Eq. (14) into Eqs. (11)-(13) and integrating over the center-of-mass momentum, the Compton tensor of Eq. (9) can be cast into the form

$$
\begin{aligned}
T^{\mu \nu}\left(q^{\prime}, q, \vec{p}\right)= & \sum_{X}\left\langle 0^{\prime}\left|J^{\mu}\left(-\vec{q}^{\prime}, 2 \vec{p}_{f}+\vec{q}^{\prime}\right)\right| X\right\rangle \frac{1}{E_{f}\left(\vec{p}_{f}\right)+\omega^{\prime}-E_{X}\left(\vec{p}_{f}+\vec{q}^{\prime}\right)}\left\langle X\left|J^{\nu}\left(\vec{q}, 2 \vec{p}_{i}+\vec{q}\right)\right| 0\right\rangle \\
& +\sum_{X}\left\langle 0^{\prime}\left|J^{\nu}\left(\vec{q}, 2 \vec{p}_{f}-\vec{q}\right)\right| X\right\rangle \frac{1}{E_{f}\left(\vec{p}_{f}\right)-\omega-E_{X}\left(\vec{p}_{f}-\vec{q}\right)}\left\langle X\left|J^{\mu}\left(-\vec{q}^{\prime}, 2 \vec{p}_{i}-\vec{q}^{\prime}\right)\right| 0\right\rangle, \\
S^{\mu 0}= & S^{0 \nu}=0, \quad S^{i j}=\delta_{i j}\left\langle 0^{\prime}\left|\sum_{\alpha} \frac{e_{\alpha}^{2}}{m_{\alpha}} \mathrm{e}^{i\left(\vec{q}-\vec{q}^{\prime}\right) \cdot \vec{r}_{\alpha}^{\prime}}\right| 0\right\rangle .
\end{aligned}
$$

\footnotetext{
${ }^{5}$ In the following we omit the subscript $f i$.
} 
Before calculating the GPs, we split the Compton tensor into two parts that are separately gauge invariant and symmetrical under photon crossing,

$$
M^{\mu \nu}=\tilde{M}_{P}^{\mu \nu}+\tilde{M}_{R}^{\mu \nu} .
$$

The modified pole term $\tilde{M}_{P}^{\mu \nu}$ is defined as

$$
\tilde{M}_{P}^{\mu \nu}=T_{P}^{\mu \nu}+G^{\mu \nu}
$$

where the pole term $T_{P}^{\mu \nu}$ corresponds to the contribution of the intermediate ground state in the direct and crossed channels and $G^{\mu \nu}$ reads

$$
G^{\mu 0}\left(q^{\prime}, q\right)=G^{0 \nu}\left(q^{\prime}, q\right)=0, \quad G^{i j}\left(q^{\prime}, q\right)=\delta_{i j} \frac{1}{M}\left\langle 0^{\prime}\left|\rho\left(-\vec{q}^{\prime}\right)\right| 0^{\prime}\right\rangle\langle 0|\rho(\vec{q})| 0\rangle \delta_{M_{f} M_{i}} .
$$

A derivation of $G^{\mu \nu}$ is given in Appendix A. In particular, $\tilde{M}_{P}^{\mu \nu}$ generates the correct Thomson amplitude in the limit $q, q^{\prime} \rightarrow 0$.

The residual term $\tilde{M}_{R}^{\mu \nu}$ is then given by

$$
\tilde{M}_{R}^{\mu \nu}=T_{R}^{\mu \nu}+\tilde{S}^{\mu \nu}
$$

where $T_{R}^{\mu \nu}$ is the contribution of the excited states in the direct and crossed channels, while the modified seagull term is $\tilde{S}^{\mu \nu}=S^{\mu \nu}-G^{\mu \nu}$.

\section{RESIDUAL COMPTON TENSOR AND GENERALIZED POLARIZABILITIES}

In this section we review the multipole expansion of the residual tensor and define the GPs according to Ref. [30]. In this context, we generalize the discussion of Ref. [30] by allowing for a general spin $I$ of the initial and final states, respectively. We then discuss two different schemes of evaluating the residual term $\tilde{M}_{R}^{\mu \nu}$, the first one based on the presentation of Ref. [37] and the second one on a systematic $1 / M$ expansion, which is capable of incorporating the constraints of photon-crossing symmetry to leading order in $M^{-1}$.

\section{A. Multipole expansion and generalized polarizabilities}

The starting point of Ref. [30] for the definition of the GPs is the multipole decomposition of the residual term, which is to be evaluated in the center-of-mass frame defined by $\vec{p}_{i}+\vec{q}=\vec{p}_{f}+\vec{q}^{\prime}=0$, 向

$$
\begin{aligned}
& \tilde{M}_{R}^{\mu \nu}\left(M_{f}, \omega^{\prime}, \vec{q}^{\prime} ; M_{i}, \omega, \vec{q}\right)= \\
& 4 \pi \sum_{\substack{\rho, L, M, \rho^{\prime}, L^{\prime}, M^{\prime}}} g_{\rho^{\prime} \rho^{\prime}} V^{\mu}\left(\rho^{\prime} L^{\prime} M^{\prime}, \hat{q}^{\prime}\right) H_{R}^{\left(\rho^{\prime} L^{\prime} M^{\prime}, \rho L M\right)}\left(M_{f}, \omega^{\prime},\left|\vec{q}^{\prime}\right| ; M_{i}, \omega,|\vec{q}|\right) V^{\nu *}(\rho L M, \hat{q}) g_{\rho \rho},
\end{aligned}
$$

\footnotetext{
${ }^{6}$ We repeat that $\omega$ and $\omega^{\prime}$ are related by energy conservation.
} 
where $\left\{V^{\mu}(\rho L M, \hat{q})\right\}$ constitutes the four-dimensional basis of the multipole expansion of four-vector fields as introduced in Appendix $\mathrm{C}$ of Ref. [30]. In particular, $\rho\left(\rho^{\prime}\right)$ denotes the type of the initial (final) multipolarity ( $\rho=0$ scalar, $\rho=1$ magnetic, $\rho=2$ electric, and $\rho=3$ longitudinal) and $L, M\left(L^{\prime}, M^{\prime}\right)$ refer to the quantum numbers of the total angular momentum of the initial (final) photon. Note that in Eq. (27) the dependence on the arguments $\vec{q}$ and $\vec{q}^{\prime}$ also results from the dependence of $\tilde{M}_{R}^{\mu \nu}$ of Eq. (26) on $\vec{p}$, given by $\vec{p}=-\left(\vec{q}+\vec{q}^{\prime}\right) / 2$ in the center-of-mass frame. This implies that a naive substitution $[\mu, \omega, \vec{q}] \leftrightarrow\left[\nu,-\omega^{\prime},-\vec{q}^{\prime}\right]$ in Eq. (27) is no longer equivalent to photon crossing, which assumes that $\vec{p}$ is not affected by such a transformation. This can also be seen from energy conservation, where "naive" photon crossing would imply

$$
\omega^{\prime}=\omega+E(-\vec{q})-E\left(-\vec{q}^{\prime}\right) \mapsto-\omega^{\prime}+E\left(\vec{q}^{\prime}\right)-E(\vec{q})
$$

which clearly contradicts the correct relation under crossing, $\omega^{\prime} \leftrightarrow-\omega$. We will come back to this point in subsection III.C.

Using the orthogonality property of the basis vectors $V^{\mu}(\rho L M, \hat{q})$, the multipoles can be extracted from Eq. (27) as

$$
\begin{aligned}
& H_{R}^{\left(\rho^{\prime} L^{\prime} M^{\prime}, \rho L M\right)}\left(M_{f}, \omega^{\prime},\left|\vec{q}^{\prime}\right| ; M_{i}, \omega,|\vec{q}|\right)= \\
& \frac{1}{4 \pi} \int \mathrm{d} \hat{q} \mathrm{~d} \hat{q}^{\prime} V_{\mu}^{*}\left(\rho^{\prime} L^{\prime} M^{\prime}, \hat{q}^{\prime}\right) \tilde{M}_{R}^{\mu \nu}\left(M_{f}, \omega^{\prime}, \vec{q}^{\prime} ; M_{i}, \omega, \vec{q}\right) V_{\nu}(\rho L M, \hat{q}) .
\end{aligned}
$$

Since the residual term is gauge invariant, $q_{\mu}^{\prime} \tilde{M}_{R}^{\mu \nu}=0=q_{\nu} \tilde{M}_{R}^{\mu \nu}$, it is sufficient to consider scalar, magnetic, and electric multipoles only [30,

$\tilde{M}_{R}^{\mu \nu}\left(M_{f}, \omega^{\prime}, \vec{q}^{\prime} ; M_{i}, \omega, \vec{q}\right)=$
$4 \pi \sum_{\rho, \rho^{\prime}=0}^{2} \sum_{\substack{L, M, L^{\prime}, M^{\prime}}} g_{\rho^{\prime} \rho^{\prime}} W^{\mu}\left(\rho^{\prime} L^{\prime} M^{\prime}, \hat{q}^{\prime}\right) H_{R}^{\left(\rho^{\prime} L^{\prime} M^{\prime}, \rho L M\right)}\left(M_{f}, \omega^{\prime},\left|\vec{q}^{\prime}\right| ; M_{i}, \omega,|\vec{q}|\right) W^{\nu *}(\rho L M, \hat{q}) g_{\rho \rho}$,

where

$$
W^{\mu}(\rho L M, \hat{q})=V^{\mu}(\rho L M, \hat{q})+\delta_{\rho 0} \frac{\omega}{|\vec{q}|} V^{\mu}(3 L M, \hat{q}), \quad \rho=0,1,2 .
$$

Finally, the dependence on the target spin projections is extracted by defining reduced multipoles, I]

$$
\begin{aligned}
H_{R}^{\left(\rho^{\prime} L^{\prime}, \rho L\right) S}\left(\omega^{\prime},\left|\vec{q}^{\prime}\right| ; \omega,|\vec{q}|\right)= & \sum_{M_{i}, M_{f}} \sum_{M, M^{\prime}}(-1)^{L+M+I+M_{f}}\left\langle I-M_{f}, I M_{i} \mid S s\right\rangle \\
& \times\left\langle L^{\prime} M^{\prime}, L-M \mid S s\right\rangle H_{R}^{\left(\rho^{\prime} L^{\prime} M^{\prime}, \rho L M\right)}\left(M_{f}, \omega^{\prime},\left|\vec{q}^{\prime}\right| ; M_{i}, \omega,|\vec{q}|\right),
\end{aligned}
$$

\footnotetext{
${ }^{7}$ The right-hand side of Eq. (65) of Ref. [30] contains a typographic error and should include a summation over the projections $s$ [56].
} 
where $I$ is the spin of the target, and any of the $2 S+1$ projections $s$ can be chosen. The selection rules due to the conservation of total angular momentum and parity are

$$
\left|L-L^{\prime}\right| \leq S \leq L+L^{\prime}, \quad 0 \leq S \leq 2 I, \quad(-1)^{\delta_{\rho 1}+L}=(-1)^{\delta_{\rho^{\prime} 1}+L^{\prime}}
$$

From now on we assume the final-state photon to be real, $\omega^{\prime}=\left|\vec{q}^{\prime}\right|$. The definition of the GPs requires to identify the leading-order behavior in $\left|\vec{q}^{\prime}\right|$ for any given multipole. In the Siegert limit of $\left|\vec{q}^{\prime}\right| \rightarrow 0$, the final-state electric and longitudinal multipoles are related by

$$
\lim _{\left|\vec{q}^{\prime}\right| \rightarrow 0} H_{R}^{\left(2 L^{\prime}, \rho L\right) S}=\lim _{\left|\vec{q}^{\prime}\right| \rightarrow 0} \sqrt{\frac{L^{\prime}+1}{L^{\prime}}} H_{R}^{\left(3 L^{\prime}, \rho L\right) S},
$$

which in turn can be expressed in terms of $H_{R}^{\left(0 L^{\prime}, \rho L\right) S}$ by gauge invariance. The leadingorder behavior of a scalar or magnetic multipole of order $L^{\prime}$ is given by $\left|\vec{q}^{\prime}\right|^{L^{\prime}}$. The treatment of the initial-state photon is similar, except that one is interested in the behavior of the multipole for arbitrary values of $|\vec{q}|$. The general relation between electric and longitudinal multipoles is given by

$$
\overrightarrow{\mathcal{E}}_{M}^{L}(\hat{q})=\overrightarrow{\mathcal{L}}_{M}^{L}(\hat{q})+\sqrt{\frac{2 L+1}{L}} \overrightarrow{\mathcal{Y}}_{M}^{L}{ }^{L+1}(\hat{q}),
$$

where $\overrightarrow{\mathcal{E}}_{M}^{L}(\hat{q}), \overrightarrow{\mathcal{L}}_{M}^{L}(\hat{q})$, and $\overrightarrow{\mathcal{Y}}_{M}^{J L}(\hat{q})$ are electric and longitudinal vectors of the multipole expansion, and vector spherical harmonics, respectively. While the first term on the righthand side of Eq. (32) can be expressed by the scalar multipole, the second term leads to the so-called mixed multipoles, which are neither of electric nor longitudinal type [30],

$$
\begin{aligned}
& \hat{H}_{R}^{\left(\rho^{\prime} L^{\prime} M^{\prime}, L M\right)}\left(M_{f}, \omega^{\prime},\left|\vec{q}^{\prime}\right| ; M_{i}, \omega,|\vec{q}|\right)= \\
& \frac{1}{4 \pi} \int \mathrm{d} \hat{q} \mathrm{~d} \hat{q}^{\prime} V_{\mu}^{*}\left(\rho^{\prime} L^{\prime} M^{\prime}, \hat{q}^{\prime}\right) \tilde{M}_{R}^{\mu i}\left(M_{f}, \omega^{\prime}, \vec{q}^{\prime} ; M_{i}, \omega,|\vec{q}|\right)\left[\overrightarrow{\mathcal{Y}}_{M}^{L L+1}(\hat{q})\right]^{i},
\end{aligned}
$$

with reduced multipoles analogous to Eq. (30]). The GPs of Ref. [30] are now defined as

$$
\begin{gathered}
P^{\left(\rho^{\prime} L^{\prime}, \rho L\right) S}(|\vec{q}|)=\left[\frac{1}{\omega^{\prime L^{\prime}}|\vec{q}|^{L}} H_{R}^{\left(\rho^{\prime} L^{\prime}, \rho L\right) S}\left(\omega^{\prime},|\vec{q}|\right)\right]_{\omega^{\prime}=0} \quad\left(\rho, \rho^{\prime}=0,1\right), \\
\hat{P}^{\left(\rho^{\prime} L^{\prime}, L\right) S}(|\vec{q}|)=\left[\frac{1}{\omega^{\prime L^{\prime}}|\vec{q}|^{L+1}} \hat{H}_{R}^{\left(\rho^{\prime} L^{\prime}, L\right) S}\left(\omega^{\prime},|\vec{q}|\right)\right]_{\omega^{\prime}=0} \quad\left(\rho^{\prime}=0,1\right) .
\end{gathered}
$$

Up to this point we have only incorporated constraints due to rotational symmetry, gauge invariance, and parity conservation. As such, the above analysis is valid in both a nonrelativistic and a relativistic framework. However, additional restrictions apply, once a covariant, field-theoretical approach is chosen. In particular, if one assumes symmetry under particle crossing in combination with charge-conjugation invariance, one obtains a set of four independent linear equations, involving the ten generalized polarizabilities with $L^{\prime}=1$ 34, 35. 


\section{B. Expansion of Liu, Thomas, and Guichon}

Let us first reconsider the calculation of the GPs according to Ref. [37], where the hadronic tensor $\tilde{M}_{R}^{\mu \nu}$ is split into a leading and a recoil contribution. For that purpose one defines the matrix elements of the charge density and current operators of Eqs. (14)(17) between the ground state and the intermediate states $X \neq 0$,

$$
\begin{aligned}
\rho_{X}(\vec{q}) & =\sum_{\alpha} e_{\alpha}\left\langle X\left|e^{i \vec{q} \cdot \vec{r}_{\alpha}^{\prime}}\right| 0\right\rangle, \\
\vec{P}_{X}(\vec{q}) & =\sum_{\alpha} \frac{e_{\alpha}}{2 m_{\alpha}}\left\langle X\left|\left\{e^{i \vec{q} \cdot \vec{r}_{\alpha}^{\prime}}, \vec{p}_{\alpha}^{\prime}\right\}\right| 0\right\rangle, \\
\vec{\Sigma}_{X}(\vec{q}) & =\sum_{\alpha} \frac{e_{\alpha}}{2 m_{\alpha}}\left\langle X\left|\vec{\sigma}_{\alpha} e^{i \vec{q} \cdot \vec{r}_{\alpha}^{\prime}}\right| 0\right\rangle .
\end{aligned}
$$

With this convention the current matrix elements of the direct-channel (subscript $d$ ) of Eq. (21) readi

$$
\begin{aligned}
& J_{d, X 0}^{0}(\vec{q}) \equiv\langle X|\rho(\vec{q})| 0\rangle=\rho_{X}(\vec{q}) \\
& \vec{J}_{d, X 0}(\vec{q}) \equiv\langle X|\vec{J}(\vec{q},-\vec{q})| 0\rangle=\vec{P}_{X}(\vec{q})+i \vec{\Sigma}_{X}(\vec{q}) \times \vec{q}-\frac{\vec{q}}{2 M} \rho_{X}(\vec{q}), \\
& J_{d, 0 X}^{0}\left(\vec{q}^{\prime}\right) \equiv\left\langle 0\left|\rho\left(-\vec{q}^{\prime}\right)\right| X\right\rangle=\rho_{X}^{*}\left(\vec{q}^{\prime}\right), \\
& \vec{J}_{d, 0 X}\left(\vec{q}^{\prime}\right) \equiv\left\langle 0\left|\vec{J}\left(-\vec{q}^{\prime},-\vec{q}^{\prime}\right)\right| X\right\rangle=\vec{P}_{X}^{*}\left(\vec{q}^{\prime}\right)-i \vec{\Sigma}_{X}^{*}\left(\vec{q}^{\prime}\right) \times \vec{q}^{\prime}-\frac{\vec{q}^{\prime}}{2 M} \rho_{X}^{*}\left(\vec{q}^{\prime}\right) .
\end{aligned}
$$

In the crossed channel (subscript $c$ ) the intermediate states propagate with momentum $\vec{p}_{X}=-\vec{q}-\vec{q}^{\prime}$, resulting in matrix elements depending on both $\vec{q}$ and $\vec{q}^{\prime}$. As in Ref. [37], we write these matrix elements as

$$
\begin{aligned}
\left\langle X\left|\vec{J}\left(-\vec{q}^{\prime},-2 \vec{q}-\vec{q}^{\prime}\right)\right| 0\right\rangle & \equiv \vec{J}_{c, X 0}\left(\vec{q}^{\prime}\right)+\delta \vec{J}_{c, X 0}\left(\vec{q}, \vec{q}^{\prime}\right), \\
\left\langle 0\left|\vec{J}\left(\vec{q},-2 \vec{q}^{\prime}-\vec{q}\right)\right| X\right\rangle & \equiv \vec{J}_{c, 0 X}(\vec{q})+\delta \vec{J}_{c, 0 X}\left(\vec{q}, \vec{q}^{\prime}\right)
\end{aligned}
$$

where

$$
\begin{aligned}
J_{c, X 0}^{0}\left(\vec{q}^{\prime}\right) & =\rho_{X}\left(-\vec{q}^{\prime}\right), \\
\vec{J}_{c, X 0}\left(\vec{q}^{\prime}\right) & =\vec{P}_{X}\left(-\vec{q}^{\prime}\right)-i \vec{\Sigma}_{X}\left(-\vec{q}^{\prime}\right) \times \vec{q}^{\prime}-\frac{\vec{q}^{\prime}}{2 M} \rho_{X}\left(-\vec{q}^{\prime}\right), \\
J_{c, 0 X}^{0}(\vec{q}) & =\rho_{X}^{*}(-\vec{q}), \\
\vec{J}_{c, 0 X}(\vec{q}) & =\vec{P}_{X}^{*}(-\vec{q})+i \vec{\Sigma}_{X}^{*}(-\vec{q}) \times \vec{q}-\frac{\vec{q}}{2 M} \rho_{X}^{*}(-\vec{q}), \\
\delta J_{c, X 0}^{0} & =\delta J_{c, 0 X}^{0}=0, \\
\delta \vec{J}_{c, X 0}\left(\vec{q}, \vec{q}^{\prime}\right) & =-\frac{\vec{q}}{M} \rho_{X}\left(-\vec{q}^{\prime}\right), \\
\delta \vec{J}_{c, 0 X}\left(\vec{q}, \vec{q}^{\prime}\right) & =-\frac{\vec{q}^{\prime}}{M} \rho_{X}^{*}(-\vec{q}) .
\end{aligned}
$$

\footnotetext{
${ }^{8}$ In the following we suppress the spin indices in our notation.
} 
In Ref. [37], the leading part of the hadronic tensor is obtained by neglecting the $\delta \vec{J}_{c}$ terms of Eqs. (38) in the crossed-channel matrix elements and by only taking account of the leading terms in the expansion of the denominators in a power series in $\omega^{\prime}$, f

$$
\begin{aligned}
\frac{1}{E_{f}\left(-\vec{q}^{\prime}\right)+\omega^{\prime}-E_{X}(\overrightarrow{0})}= & \frac{-1}{\Delta E_{X}}\left[1+\frac{\omega^{\prime}}{\Delta E_{X}}+\mathcal{O}\left(\omega^{\prime 2}\right)\right] \\
\frac{1}{E_{i}(-\vec{q})-E_{X}\left(-\vec{q}-\vec{q}^{\prime}\right)-\omega^{\prime}}= & \frac{1}{E_{i}(\vec{q})-E_{X}(\vec{q})}\left[1+\frac{1}{E_{i}(\vec{q})-E_{X}(\vec{q})}\left(\omega^{\prime}+\frac{\vec{q} \cdot \vec{q}^{\prime}}{E_{X}(\vec{q})}\right)\right] \\
& +\mathcal{O}\left(\omega^{\prime 2}\right),
\end{aligned}
$$

where $\Delta E_{X}$ is the excitation energy of the state $X$. As a result one finds [see Eq. (20) of Ref. [37]

$$
\tilde{M}_{R-L E A D I N G}^{\mu \nu}\left(\vec{q}^{\prime}, \vec{q}\right)=-\sum_{X \neq 0}\left[\frac{J_{d, 0 X}^{\mu}\left(\vec{q}^{\prime}\right) J_{d, X 0}^{\nu}(\vec{q})}{\Delta E_{X}}\right]-\sum_{X \neq 0}\left[\frac{J_{c, 0 X}^{\nu}(\vec{q}) J_{c, X 0}^{\mu}\left(\vec{q}^{\prime}\right)}{E_{X}(\vec{q})-E_{i}(\vec{q})}\right]+\tilde{S}^{\mu \nu}
$$

The remaining contribution from the crossed channel is included in the recoil part, which also contains additional terms of first order in $\omega^{\prime}$ from the expansion of the energy denominators in Eqs. (39). Accordingly, we obtain for the recoil part

$$
\begin{aligned}
& \tilde{M}_{R-R E C O I L}^{\mu \nu}\left(\vec{q}^{\prime}, \vec{q}\right)= \\
& -\sum_{X \neq 0} \frac{\omega^{\prime}}{\left(\Delta E_{X}\right)^{2}} J_{d, 0 X}^{\mu}\left(\vec{q}^{\prime}\right) J_{d, X 0}^{\nu}(\vec{q}) \\
& +\sum_{X \neq 0} \frac{1}{\left[E_{X}(\vec{q})-E_{i}(\vec{q})\right]^{2}}\left[\omega^{\prime}+\frac{\vec{q} \cdot \vec{q}^{\prime}}{E_{X}(\vec{q})}\right] J_{c, 0 X}^{\nu}(\vec{q})\left[J_{c, X 0}^{\mu}\left(\vec{q}^{\prime}\right)+\delta J_{c, X 0}^{\mu}\left(\vec{q}, \vec{q}^{\prime}\right)\right] \\
& -\sum_{X \neq 0} \frac{1}{E_{X}(\vec{q})-E_{i}(\vec{q})}\left[J_{c, 0 X}^{\nu}(\vec{q}) \delta J_{c, X 0}^{\mu}\left(\vec{q}, \vec{q}^{\prime}\right)+\delta J_{c, 0 X}^{\nu}\left(\vec{q}, \vec{q}^{\prime}\right) J_{c, X 0}^{\mu}\left(\vec{q}^{\prime}\right)+\delta J_{c, 0 X}^{\nu}\left(\vec{q}, \vec{q}^{\prime}\right) \delta J_{c, X 0}^{\mu}\left(\vec{q}, \vec{q}^{\prime}\right)\right] \\
& +\mathcal{O}\left(\omega^{\prime 2}\right) .
\end{aligned}
$$

This result differs from Eq. (21) of Ref. [37] by the presence of the first sum on the righthand side, the $\delta J$ term of the second sum, and the $\delta J \delta J$ of the third sum of Eq. (41). However, this difference has no bearing on the calculation of the ten GPs with $L^{\prime}=1$. In this case we only need to analyze terms of Eq. (41) that are linear in $\omega^{\prime}=\left|\vec{q}^{\prime}\right|$ [see also Eqs. (34)]. Furthermore, it follows from Eqs. (28) and (33) that the projection involves angular integrals of the type $\int \mathrm{d} \hat{q}^{\prime} Y_{1 M^{\prime}}^{*}\left(\hat{q}^{\prime}\right) \tilde{M}_{R}^{0 \nu}$ and $\int \mathrm{d} \hat{q}^{\prime} Y_{1 m^{\prime}}^{*}\left(\hat{q}^{\prime}\right) \tilde{M}_{R}^{i \nu}$ for scalar and magnetic final dipole radiation, respectively. The recoil contribution of Eq. (41) only modifies the GPs with a magnetic photon in the final state, as observed in Ref. [37]. In fact, for $\mu=0$ we need to consider $J_{d, 0 X}^{0}, J_{c, X 0}^{0}$, and $\delta J_{c, X 0}^{0}$. According to Eq. (38), the last term vanishes

\footnotetext{
${ }^{9}$ In a nonrelativistic framework consistent with Eq. (1) one would have $E_{i}(\vec{q})-E_{X}(\vec{q})=$ $-\Delta E_{X}$. However, in Ref. [37 the relativistic expressions $E_{i}(\vec{q})=\sqrt{M^{2}+\vec{q}^{2}}$ and $E_{X}(\vec{q})=$ $\sqrt{\left(M+\Delta E_{X}\right)^{2}+\vec{q}^{2}}$ have been used.
} 
identically. The first two terms have to be always evaluated at $\vec{q}^{\prime}=0$, because they are multiplied by expressions that are either manifestly or implicitly $\left[\delta \vec{J}_{c, 0 X}\left(\vec{q}, \vec{q}^{\prime}\right)\right]$ of order $\omega^{\prime}$. From Eqs. (36) and (38) we see that the corresponding overlap integrals vanish due to the orthogonality of the excited states with respect to the ground state. In conclusion, the $L^{\prime}=1$ scalar GPs do not receive a contribution from Eq. (41). For the $L^{\prime}=1$ magnetic GPs we need to consider $\mu \neq 0$. In this case the terms proportional to $\omega^{\prime}$ do not contribute, because the angular integral of $Y_{1 m^{\prime}}^{*}$ multiplied by a $\vec{q}^{\prime}$-independent function vanishes. Furthermore, since $\delta \vec{J}_{c, 0 X}\left(\vec{q}, \vec{q}^{\prime}\right)$ is of order $\omega^{\prime}$, the last term in Eq. (41) containing $\delta \vec{J}_{c, X 0}(\vec{q}, \overrightarrow{0})$ vanishes due to orthogonality. In conclusion, one is left with

$$
\begin{aligned}
\tilde{M}_{R-R E C O I L}^{\mu \nu}\left(\vec{q}^{\prime}, \vec{q}\right)= & \sum_{X \neq 0} \frac{1}{\left[E_{X}(\vec{q})-E_{i}(\vec{q})\right]^{2}} \frac{\vec{q} \cdot \vec{q}^{\prime}}{E_{X}(\vec{q})} J_{c, 0 X}^{\nu}(\vec{q}) J_{c, X 0}^{\mu}\left(\vec{q}^{\prime}\right) \\
& -\sum_{X \neq 0} \frac{1}{E_{X}(\vec{q})-E_{i}(\vec{q})}\left[J_{c, 0 X}^{\nu}(\vec{q}) \delta J_{c, X 0}^{\mu}\left(\vec{q}, \vec{q}^{\prime}\right)+\delta J_{c, 0 X}^{\nu}\left(\vec{q}, \vec{q}^{\prime}\right) J_{c, X 0}^{\mu}\left(\vec{q}^{\prime}\right)\right] \\
& +\cdots+\mathcal{O}\left(\omega^{\prime 2}\right),
\end{aligned}
$$

where the ellipses denote terms which do not contribute to the $L^{\prime}=1$ GPs.

\section{Nonrelativistic $1 / M$ expansion}

In this subsection we will consider a different procedure of ordering the contributions of the excited states to the Compton tensor. Our aim is to analyze the implications of photon-crossing symmetry for the GPs. We will see that some of the GPs will have to satisfy certain conditions for $|\vec{q}| \rightarrow 0$. At first, we will not specify the reference frame but both photons will be allowed to be virtual [see Eq. (21) for $X \neq 0$ ]. Only at the end we will assume the initial and final photons to be virtual and real, respectively, and restrict ourselves to the center-of-mass frame.

The starting point is a separation of four-current matrix elements into an intrinsic current with respect to the center-of-mass system and a center-of-mass convection current [53] [see Eqs. (14)-(17)]. As an example, let us consider the following matrix element entering the direct channel

$$
\left\langle 0\left|J^{\mu}\left(-\vec{q}^{\prime}, 2 \vec{p}+\vec{q}\right)\right| X\right\rangle=\left\langle 0\left|J^{\mu}\left(-\vec{q}^{\prime}, 0\right)\right| X\right\rangle+\left\langle 0\left|\delta J^{\mu}\left(-\vec{q}^{\prime}, 2 \vec{p}+\vec{q}\right)\right| X\right\rangle,
$$

with analogous expressions for the other combinations. Here, we have defined for generic momenta $\vec{a}$ and $\vec{b}$

$$
\begin{aligned}
& \mu=0:\left\langle 0\left|\delta J^{0}(\vec{a}, \vec{b})\right| X\right\rangle=0 \\
& \mu \neq 0:\langle 0|\delta \vec{J}(\vec{a}, \vec{b})| X\rangle=\frac{\vec{b}}{2 M}\langle 0|\rho(\vec{a})| X\rangle
\end{aligned}
$$

Using nonrelativistic kinematics $T(\vec{p})=\vec{p}^{2} /(2 M)$, we separate the energy denominators into excitation pieces and kinetic contributions, 


$$
\begin{aligned}
\frac{1}{E_{f}\left(\vec{p}_{f}\right)+\omega^{\prime}-E_{X}\left(\vec{p}_{f}+\vec{q}^{\prime}\right)} & =-\frac{1}{\Delta E_{X}}\left[1+\frac{\omega^{\prime}}{\Delta E_{X}}+\frac{T\left(\vec{p}_{f}\right)-T\left(\vec{p}_{f}+\vec{q}^{\prime}\right)}{\Delta E_{X}}+\frac{\omega^{\prime 2}}{\left(\Delta E_{X}\right)^{2}}+\mathcal{O}(3)\right], \\
\frac{1}{E_{f}\left(\vec{p}_{f}\right)-\omega-E_{X}\left(\vec{p}_{f}-\vec{q}\right)} & =-\frac{1}{\Delta E_{X}}\left[1-\frac{\omega}{\Delta E_{X}}+\frac{T\left(\vec{p}_{f}\right)-T\left(\vec{p}_{f}-\vec{q}\right)}{\Delta E_{X}}+\frac{\omega^{2}}{\left(\Delta E_{X}\right)^{2}}+\mathcal{O}(3)\right]
\end{aligned}
$$

where $\mathcal{O}(3)$ refers to terms which are suppressed by $1 /\left(\Delta E_{X}\right)^{3}, 1 /\left[\left(\Delta E_{X}\right)^{2} M\right]$, etc. In this representation we have

$$
\begin{aligned}
& T_{f i ; X}^{\mu \nu}\left(q^{\prime}, q, \vec{p}\right)= \\
& -\sum_{X \neq 0} \frac{\left[\left\langle 0\left|J^{\mu}\left(-\vec{q}^{\prime}, 0\right)\right| X\right\rangle+\left\langle 0\left|\delta J^{\mu}\left(-\vec{q}^{\prime}, 2 \vec{p}+\vec{q}\right)\right| X\right\rangle\right]\left[\left\langle X\left|J^{\nu}(\vec{q}, 0)\right| 0\right\rangle+\left\langle X\left|\delta J^{\nu}\left(\vec{q}, 2 \vec{p}+\vec{q}^{\prime}\right)\right| 0\right\rangle\right]}{\Delta E_{X}} \\
& \quad \times\left[1+\frac{\omega^{\prime}}{\Delta E_{X}}+\frac{T\left(\vec{p}_{f}\right)-T\left(\vec{p}_{f}+\vec{q}^{\prime}\right)}{\Delta E_{X}}+\frac{\omega^{\prime 2}}{\left(\Delta E_{X}\right)^{2}}+\mathcal{O}(3)\right] \\
& -\sum_{X \neq 0} \frac{\left[\left\langle 0\left|J^{\nu}(\vec{q}, 0)\right| X\right\rangle+\left\langle 0\left|\delta J^{\nu}\left(\vec{q}, 2 \vec{p}-\vec{q}^{\prime}\right)\right| X\right\rangle\right]\left[\left\langle X\left|J^{\mu}\left(-\vec{q}^{\prime}, 0\right)\right| 0\right\rangle+\left\langle X\left|\delta J^{\mu}\left(-\vec{q}^{\prime}, 2 \vec{p}-\vec{q}\right)\right| 0\right\rangle\right]}{\Delta E_{X}} \\
& \quad \times\left[1-\frac{\omega}{\Delta E_{X}}+\frac{T\left(\vec{p}_{f}\right)-T\left(\vec{p}_{f}-\vec{q}\right)}{\Delta E_{X}}+\frac{\omega^{2}}{\left(\Delta E_{X}\right)^{2}}+\mathcal{O}(3)\right] .
\end{aligned}
$$

In order to expand Eq. (43) in powers of $1 / M$, we introduce the following abbreviations for the direct-channel $(d)$ terms:

$$
\begin{aligned}
A_{d}^{\nu} & =\left\langle X\left|J^{\nu}(\vec{q}, 0)\right| 0\right\rangle, \\
\delta A_{d}^{\nu} & =\left\langle X\left|\delta J^{\nu}\left(\vec{q}, 2 \vec{p}+\vec{q}^{\prime}\right)\right| 0\right\rangle, \\
B_{d}^{\mu} & =\left\langle 0\left|J^{\mu}\left(-\vec{q}^{\prime}, 0\right)\right| X\right\rangle, \\
\delta B_{d}^{\mu} & =\left\langle 0\left|\delta J^{\mu}\left(-\vec{q}^{\prime}, 2 \vec{p}+\vec{q}\right)\right| X\right\rangle, \\
\Delta T_{d} & =T\left(\vec{p}_{f}\right)-T\left(\vec{p}_{f}+\vec{q}^{\prime}\right) .
\end{aligned}
$$

The corresponding expressions for the the crossed channel $(c)$ are obtained by simply replacing $\vec{q} \leftrightarrow-\vec{q}^{\prime}$ and $\mu \leftrightarrow \nu$. We note that both $\delta$ and $\Delta$ terms count as order $1 / M$.

The terms of leading order in $1 / M$ and arbitrary order in $1 / \Delta E_{X}$,

$$
T_{f i ; X, l . o .}^{\mu \nu}\left(q^{\prime}, q\right)=-\sum_{X \neq 0}\left(\frac{B_{d}^{\mu} A_{d}^{\nu}}{\Delta E_{X}-\omega^{\prime}}+\frac{B_{c}^{\nu} A_{c}^{\mu}}{\Delta E_{X}+\omega}\right),
$$

do not depend on $\vec{p}$. Equation (44) is symmetric under "true photon crossing," but also under "naive photon crossing" in any frame, because it is independent of $\vec{p}$. As is shown in App. B, this property implies the following conditions on the leading behavior in $1 / M$ for the reduced multipoles

$$
H_{R, l . o .}^{\left(\rho^{\prime} L^{\prime}, \rho L\right) S}\left(\omega^{\prime},\left|\vec{q}^{\prime}\right| ; \omega,|\vec{q}|\right)=g_{\rho^{\prime} \rho^{\prime}} g_{\rho \rho}(-)^{L+L^{\prime}-S} H_{R, l . o .}^{\left(\rho L, \rho^{\prime} L^{\prime}\right) S}\left(-\omega,|\vec{q}| ;-\omega^{\prime},\left|\vec{q}^{\prime}\right|\right) .
$$

Next we consider the $1 / M$ corrections to Eq. (44) to arbitrary order in $1 / \Delta E_{X}$ : 


$$
\begin{aligned}
-\sum_{X \neq 0} & {\left[\frac{B_{d}^{\mu} \delta A_{d}^{\nu}+\delta B_{d}^{\mu} A_{d}^{\nu}}{\Delta E_{X}-\omega^{\prime}}+\frac{B_{c}^{\nu} \delta A_{c}^{\mu}+\delta B_{c}^{\nu} A_{c}^{\mu}}{\Delta E_{X}+\omega}\right.} \\
& \left.+\frac{B_{d}^{\mu} A_{d}^{\nu}}{\Delta E_{X}-\omega^{\prime}} \frac{\Delta T_{d}}{\Delta E_{X}-\omega^{\prime}}+\frac{B_{c}^{\nu} A_{c}^{\mu}}{\Delta E_{X}+\omega} \frac{\Delta T_{c}}{\Delta E_{X}+\omega}\right] .
\end{aligned}
$$

The first line of Eq. (46) contains the modifications of the current matrix elements to first order in $1 / M$ whereas the second line involves the center-of-mass kinetic energies of the final and the intermediate states, respectively. In this scheme, each order is separately photon-crossing symmetric. However, terms beyond leading order in $1 / M$ will, in general, not be symmetric under naive photon crossing in the center-of-mass frame.

We now turn to the case of real photons in the final state, $\omega^{\prime}=\left|\vec{q}^{\prime}\right|$, and explicitly specify the center-of-mass frame. As we are not interested in terms beyond linear order in $\omega^{\prime}$ and $1 / M$, we make use of energy conservation,

$$
\omega \simeq \omega^{\prime}-\frac{\vec{q}^{2}}{2 M},
$$

to expand the crossed-channel terms as

$$
\frac{1}{\Delta E_{X}+\omega}=\frac{1}{\Delta E_{X}}\left[1-\frac{\omega^{\prime}}{\Delta E_{X}}+\frac{\vec{q}^{2}}{2 M \Delta E_{X}}-\frac{\omega^{\prime} \vec{q}^{2}}{\left(\Delta E_{X}\right)^{2} M}+\mathcal{O}\left(\omega^{\prime 2}\right)\right] .
$$

We then obtain from Eq. (44) up to and including terms linear in $\omega^{\prime}$ and $1 / M$

$$
\begin{aligned}
T_{f i ; X, l . o .}^{\mu \nu}\left(\vec{q}^{\prime}, \vec{q}\right)= & -\sum_{X \neq 0}\left[\frac{1}{\Delta E_{X}}\left(1+\frac{\omega^{\prime}}{\Delta E_{X}}\right) B_{d}^{\mu} A_{d}^{\nu}+\frac{1}{\Delta E_{X}}\left(1-\frac{\omega^{\prime}}{\Delta E_{X}}\right) B_{c}^{\nu} A_{c}^{\mu}\right. \\
& \left.+\frac{\vec{q}^{2}}{2 M\left(\Delta E_{X}\right)^{2}} B_{c}^{\nu} A_{c}^{\mu}-\frac{\omega^{\prime} \vec{q}^{2}}{\left(\Delta E_{X}\right)^{3} M} B_{c}^{\nu} A_{c}^{\mu}\right] \\
& +\mathcal{O}\left(\omega^{\prime 2}, 1 / M^{2}\right) .
\end{aligned}
$$

Note that via energy conservation, Eq. (44) has also generated terms in Eq. (47) which are of higher order in $1 / M$.

For further evaluation of Eq. (46) we use

$$
\Delta T_{d}=\mathcal{O}\left(\omega^{\prime 2}\right), \quad \Delta T_{c}=-\frac{\vec{q}^{2}}{2 M}-\frac{\vec{q} \cdot \vec{q}^{\prime}}{M},
$$

and obtain

$$
\begin{aligned}
T_{f i ; X, \text { n.l.o. }}^{\mu \nu}\left(\vec{q}^{\prime}, \vec{q}\right)= & -\sum_{X \neq 0}\left[\frac{1}{\Delta E_{X}}\left(1+\frac{\omega^{\prime}}{\Delta E_{X}}\right)\left(B_{d}^{\mu} \delta A_{d}^{\nu}+\delta B_{d}^{\mu} A_{d}^{\nu}\right)\right. \\
& +\frac{1}{\Delta E_{X}}\left(1-\frac{\omega^{\prime}}{\Delta E_{X}}\right)\left(B_{c}^{\nu} \delta A_{c}^{\mu}+\delta B_{c}^{\nu} A_{c}^{\mu}\right) \\
& \left.-\frac{\vec{q}^{2}}{2 M} B_{c}^{\nu} A_{c}^{\mu} \frac{1}{\left(\Delta E_{X}\right)^{2}}\left(1-2 \frac{\omega^{\prime}}{\Delta E_{X}}\right)-\frac{\vec{q} \cdot \vec{q}^{\prime}}{M} B_{c}^{\nu} A_{c}^{\mu} \frac{1}{\left(\Delta E_{X}\right)^{2}}\right] \\
& +\mathcal{O}\left(\omega^{\prime 2}, 1 / M^{2}\right) .
\end{aligned}
$$


The last two terms of Eq. (47) cancel with two corresponding terms in Eq. (48) and the sum can be written as

$$
\begin{aligned}
T_{f i ; X}^{\mu \nu}\left(\vec{q}^{\prime}, \vec{q}\right)= & -\sum_{X \neq 0}\left[\frac{B_{d}^{\mu} A_{d}^{\nu}}{\Delta E_{X}}+\frac{B_{c}^{\nu} A_{c}^{\mu}}{\Delta E_{X}}\right] \\
& -\sum_{X \neq 0} \frac{\omega^{\prime}}{\left(\Delta E_{X}\right)^{2}} B_{d}^{\mu} A_{d}^{\nu} \\
& +\sum_{X \neq 0} \frac{1}{\left(\Delta E_{X}\right)^{2}}\left[\omega^{\prime}+\frac{\vec{q} \cdot \vec{q}^{\prime}}{M}\right] B_{c}^{\nu} A_{c}^{\mu} \\
& -\sum_{X \neq 0}\left[\frac{B_{d}^{\mu} \delta A_{d}^{\nu}+\delta B_{d}^{\mu} A_{d}^{\nu}}{\Delta E_{X}}+\frac{B_{c}^{\nu} \delta A_{c}^{\mu}+\delta B_{c}^{\nu} A_{c}^{\mu}}{\Delta E_{X}}\right] \\
& \left.-\sum_{X \neq 0} \frac{\omega^{\prime}}{\left(\Delta E_{X}\right)^{2}}\left(B_{d}^{\mu} \delta A_{d}^{\nu}+\delta B_{d}^{\mu} A_{d}^{\nu}-B_{c}^{\nu} \delta A_{c}^{\mu}-\delta B_{c}^{\nu} A_{c}^{\mu}\right)\right] \\
& +\mathcal{O}\left(\omega^{\prime 2}, 1 / M^{2}\right) .
\end{aligned}
$$

As in the previous case we will now analyze which terms of Eq. (49) actually generate a contribution to the $L^{\prime}=1$ GPs after angular integration with the spherical harmonics $Y_{1 m^{\prime}}^{*}\left(\hat{q}^{\prime}\right)$. Again, all terms explicitly proportional to $\omega^{\prime}$ will not contribute, because at leading order they are multiplied by expressions which do not depend on the direction of $\hat{q}^{\prime}$. The $\delta B_{d}^{\mu}$ term vanishes for $\mu=0$ and is of order $\omega^{\prime 2}$ for $\mu \neq 0$, and one is left with

$$
T_{f i ; X}^{\mu \nu}\left(\vec{q}^{\prime}, \vec{q}\right)=T_{f i ; X, L E A D I N G}^{\mu \nu}\left(\vec{q}^{\prime}, \vec{q}\right)+T_{f i ; X, R E C O I L}^{\mu \nu}\left(\vec{q}^{\prime}, \vec{q}\right),
$$

where

$$
\begin{aligned}
T_{f i, X, L E A D I N G}^{\mu \nu}\left(\vec{q}^{\prime}, \vec{q}\right)= & -\sum_{X \neq 0}\left[\frac{B_{d}^{\mu} A_{d}^{\nu}}{\Delta E_{X}}+\frac{B_{c}^{\nu} A_{c}^{\mu}}{\Delta E_{X}}\right], \\
T_{f i ; X, R E C O I L}^{\mu \nu}\left(\vec{q}^{\prime}, \vec{q}\right)= & -\sum_{X \neq 0}\left[\frac{B_{d}^{\mu} \delta A_{d}^{\nu}+\delta B_{c}^{\nu} A_{c}^{\mu}}{\Delta E_{X}}\right] \\
& +\sum_{X \neq 0} \frac{1}{\left(\Delta E_{X}\right)^{2}} \frac{\vec{q} \cdot \vec{q}^{\prime}}{M} B_{c}^{\nu} A_{c}^{\mu} \\
& -\sum_{X \neq 0} \frac{B_{c}^{\nu} \delta A_{c}^{\mu}}{\Delta E_{X}} \\
& +\cdots+\mathcal{O}\left(\omega^{\prime 2}, 1 / M^{2}\right),
\end{aligned}
$$

where the ellipses refer to terms which do not contribute to the $L^{\prime}=1$ GPs. The terms in Eq. (51) generate contributions to the GPs which result entirely from intrinsic currents. The first line of the recoil term in Eq. (52) corresponds to $1 / M$ corrections of the virtualphoton absorption vertices in the direct and crossed channel, respectively. The second and third lines are $1 / M$ corrections of the crossed-channel energy denominator and real-photon vertex, respectively. These last two corrections only affect GPs involving a magnetic photon in the final state. 
Finally, by taking the nonrelativistic limit of the energies in the scheme of Ref. [37],

$$
\begin{aligned}
& E_{i}(\vec{q})=\sqrt{M^{2}+\vec{q}^{2}} \simeq M+\frac{\vec{q}^{2}}{2 M}, \\
& E_{X}(\vec{q})==\sqrt{M_{X}^{2}+\vec{q}^{2}} \simeq M+\Delta E_{X}+\frac{\vec{q}^{2}}{2 M},
\end{aligned}
$$

it is straightforward to verify that the two expansion schemes coincide up to and including terms of order $\omega^{\prime}$ and $1 / M$. They only differ in their separation into leading and recoil terms.

\section{GENERALIZED POLARIZABILITIES IN A NRCQM}

In this section we discuss the GPs of the nucleon in the framework of a nonrelativistic system consisting of three constituent quarks. As in Ref. [37] we restrict ourselves to the inclusion of the $\Delta(1232)$ resonance and the low-lying negative-parity baryons $\mathrm{D}_{13}(1520)$, $\mathrm{S}_{11}(1535), \mathrm{S}_{31}(1620), \mathrm{S}_{11}(1650), \mathrm{S}_{13}(1700)$, and $\mathrm{D}_{33}(1700)$.

\section{A. Matrix elements in the Isgur-Karl model}

To be specific, we employ the model of Isgur and Karl [57] which describes the quarkquark potential by a harmonic-oscillator term plus a spin-dependent hyperfine interaction. The baryon states are expressed in a basis of $\mathrm{SU}(6)$ harmonic-oscillator wave functions, with the $\mathrm{SU}(6)$ multiplets generated by the combination of $\mathrm{SU}(2)_{\text {spin }}$ and $\mathrm{SU}(3)_{\text {flavor }}$ multiplets. In particular, the nucleon and the $\Delta(1232)$ resonance belong to the groundstate spin- $1 / 2$ octet, ${ }^{2} 8$, and the spin- $3 / 2$ decuplet, ${ }^{4} 10$, of the $\underline{56} \mathrm{SU}(6)$ supermultiplet, respectively. The multiplet of states associated with the negative-parity orbital excitation is classified in terms of a $\underline{70}$ supermultiplet of $\mathrm{SU}(6)$ which decomposes into ${ }^{2} 1,{ }^{2} 8,{ }^{4} 8$, and ${ }^{2} 10$ multiplets. According to Ref. [57], the strength of the hyperfine interaction is fixed to reproduce the experimental mass splitting of $N$ and $\Delta(1232)$ states, while the remaining orbital excitations of the $\underline{70}$ multiplet are constructed with mixing parameters describing the empirical spectrum quite well.

Since we are interested in the non-strange sector only, we assume that all three quarks have equal masses $m_{q}$. In addition, when calculating the matrix elements of the electromagnetic current of Eqs. (16) and (17), we take advantage of the overall symmetry of the $\mathrm{SU}(6)$ harmonic-oscillator wave function. This allows us to simplify the matrix elements of one-body operators,

$$
\left\langle A\left|\sum_{i=1}^{3} \hat{O}_{i}\right| B\right\rangle=3\left\langle A\left|\hat{O}_{3}\right| B\right\rangle .
$$

As a result, the overlap integrals of Eq. (35) can be written as

$$
\rho_{X}(\vec{q})=3 \int d \vec{\rho} d \vec{\lambda} \mathrm{e}^{-i \sqrt{\frac{2}{3}} \vec{q} \cdot \vec{\lambda}} \phi_{X}^{\dagger} \hat{Q} \phi_{N},
$$




$$
\begin{aligned}
& \vec{P}_{X}(\vec{q})=\sqrt{\frac{2}{3}} \frac{3}{2 m_{q}} \int d \vec{\rho} d \vec{\lambda} \mathrm{e}^{-i \sqrt{\frac{2}{3}} \vec{q} \cdot \vec{\lambda}} \phi_{X}^{\dagger}\left(i \vec{\nabla}_{\lambda}-i \overleftarrow{\nabla} \lambda\right) \hat{Q} \phi_{N}, \\
& \vec{\Sigma}_{X}(\vec{q})=\frac{3}{2 m_{q}} \int d \vec{\rho} d \vec{\lambda} \mathrm{e}^{-i \sqrt{\frac{2}{3}} \vec{q} \cdot \vec{\lambda}} \phi_{X}^{\dagger} \vec{\sigma}_{3} \hat{Q} \phi_{N},
\end{aligned}
$$

where

$$
\begin{aligned}
& \phi_{N}=\phi_{N}\left(\vec{\rho}, \vec{\lambda}, \frac{1}{2}, M, \frac{1}{2}, \tau_{N}\right), \\
& \phi_{X}=\phi_{X}\left(\vec{\rho}, \vec{\lambda}, J_{X}, M_{X}, I_{X}, \tau_{X}\right)
\end{aligned}
$$

denote the internal wave functions of the nucleon and the excited states, respectively, with an obvious notation for spin and isospin labels. We have introduced the standard Jacobi coordinates $\vec{\rho}=\left(\vec{r}_{1}-\vec{r}_{2}\right) / \sqrt{2}$ and $\vec{\lambda}=\left(\vec{r}_{1}+\vec{r}_{2}-2 \vec{r}_{3}\right) / \sqrt{6}$, and made use of $\vec{r}_{3}^{\prime}=\vec{r}_{3}-\vec{R}=-\sqrt{2 / 3} \vec{\lambda}$. Furthermore, $\hat{Q}=\left(1 / 6+\tau_{3} / 2\right)$ and $\vec{\sigma}_{3}$ denote the charge operator and the Pauli matrices of the third quark, respectively.

Explicit expressions for the contributions of the $P$-wave negative-parity states are given in Appendix $\mathbf{Q}$.

\section{B. GPs in the framework of Liu, Thomas, and Guichon}

We first discuss the results for the proton GPs that we obtain with the same conventions used by Ref. [37] for the separation into leading and recoil terms. Following Ref. [57, we use $m_{q}=350 \mathrm{MeV}$ for the quark mass and $\alpha=320 \mathrm{MeV}$ for the oscillator parameter. As was pointed out in Ref. [37], the proton polarizabilities do not receive any contribution from the $N\left({ }^{4} 8\right)$ multiplets. The mixing parameters $a_{X}$ encoding the $N\left({ }^{2} 8\right)$ and $\Delta\left({ }^{2} 10\right)$ composition of the resonant states are taken from Ref. [57] and are listed in Tab. I.

With these assumptions we find for the leading contributions to the Compton tensor of Eq. (40)

$$
\begin{aligned}
P^{(01,01) S} & =\frac{1}{18} \frac{1}{\alpha^{2}} e^{-q^{2} / 6 \alpha^{2}} \sum_{X=N^{*}, \Delta^{*}} a_{X}^{2}\left(\frac{Z_{d}^{S, J_{X}}}{M-M_{X}}+\frac{Z_{c}^{S, J_{X}}}{E(q)-E_{X}(q)}\right) \\
P^{(01,12) 1} & =\frac{1}{36} \sqrt{\frac{3}{5}} \frac{1}{m_{q} \alpha^{2}} e^{-q^{2} / 6 \alpha^{2}} \sum_{X=N^{*}, \Delta^{*}} a_{X}^{2} \frac{(-1)^{I_{x}-1 / 2}}{2 I_{x}}\left(\frac{Z_{a d}^{2, S, J_{X}}}{M-M_{X}}+\frac{Z_{a c}^{2, S, J_{X}}}{E(q)-E_{X}(q)}\right), \\
P_{\text {para }}^{(11,11) S} & =\frac{4}{27} \frac{1}{m_{q}^{2}} e^{-q^{2} / 6 \alpha^{2}}\left(\frac{Z_{\Delta}^{S}}{M-M_{\Delta}}+(-1)^{S} \frac{Z_{\Delta}^{S}}{E(q)-E_{\Delta}(q)}\right) \\
P_{\text {dia }}^{(11,11) S} & =\delta_{S 0} \frac{1}{3 \sqrt{6}} \frac{1}{m_{q} \alpha^{2}} e^{-q^{2} / 6 \alpha^{2}} .
\end{aligned}
$$

The angular coefficients $Z$ of the leading contributions are given in Tab. II. The diamagnetic term of Eq. (60) originates from the modified seagull term $\tilde{S}^{\mu \nu}$ and contributes in the spin-independent case only. The mixed GPs are given as the sum of two terms, corresponding to the contributions from the convective $\left(\hat{P}_{F}^{(01,1) S}\right)$ and spin $\left(\hat{P}_{\Sigma}^{(01,1) S}\right)$ terms of the current at the virtual-photon vertex 


$$
\begin{aligned}
& \hat{P}^{(01,1) S}=\hat{P}_{F}^{(01,1) S}+\hat{P}_{\Sigma}^{(01,1) S}, \\
& \hat{P}_{F}^{(01,1) S}=\frac{\sqrt{2}}{108 \sqrt{3}} \frac{1}{m_{q} \alpha^{2}} e^{-q^{2} / 6 \alpha^{2}} \sum_{X=N^{*}, \Delta^{*}} a_{X}^{2}\left(\frac{Z_{d}^{S, J_{X}}}{M-M_{X}}+\frac{Z_{c}^{S, J_{X}}}{E(q)-E_{X}(q)}\right), \\
& \hat{P}_{\Sigma}^{(01,1) S}=-\frac{1}{36 \sqrt{3}} \frac{1}{m_{q} \alpha^{2}} e^{-q^{2} / 6 \alpha^{2}} \sum_{X=N^{*}, \Delta^{*}} a_{X}^{2} \frac{(-1)^{I_{x}-1 / 2}}{2 I_{x}}\left(\frac{Z_{a d}^{1, S, J_{X}}}{M-M_{X}}+\frac{Z_{a c}^{1, S, J_{X}}}{E(q)-E_{X}(q)}\right) .
\end{aligned}
$$

In the scheme of Ref. [37] the spin-dependent GPs $P^{(11,02) 1}, P^{(11,00) 1}$, and $\hat{P}^{(11,2) 1}$, all of which lead to M1 radiation in the final state, vanish identically at leading order.

The recoil corrections are exclusively generated by the crossed-channel diagrams and only modify the GPs with a magnetic final photon [37] (see the discussion at the end of Sec. III.B). To start with, the two GPs $P^{(11,00) 1}$ and $P^{(11,02) 1}$ receive a non-vanishing recoil contribution

$$
\begin{aligned}
& P_{\text {recoil }}^{(11,00) 1}=-\frac{1}{\sqrt{3}} \frac{q^{2}}{m_{q}} e^{-q^{2} / 6 \alpha^{2}} \sum_{X=N^{*}, \Delta^{*}} \frac{a_{X}^{2} Z_{1100}^{J_{X}}}{E_{X}(q)\left[E(q)-E_{X}(q)\right]^{2}}\left[1-\frac{E_{X}(q)\left[E(q)-E_{X}(q)\right]}{3 \alpha^{2}}\right], \\
& P_{\text {recoil }}^{(11,02) 1}=-\frac{1}{\sqrt{3}} \frac{1}{m_{q}} e^{-q^{2} / 6 \alpha^{2}} \sum_{X=N^{*}, \Delta^{*}} \frac{a_{X}^{2} Z_{1102}^{J_{X}}}{E_{X}(q)\left[E(q)-E_{X}(q)\right]^{2}}\left[1-\frac{E_{X}(q)\left[E(q)-E_{X}(q)\right]}{3 \alpha^{2}}\right] .
\end{aligned}
$$

When discussing the recoil contribution to the remaining three polarizabilities $P_{\text {recoil }}^{(11,11) S}$ and $\hat{P}_{\text {recoil }}^{(11,2) 1}$, it is useful to distinguish between terms which result from the spinindependent $(\mathrm{C})$ and the spin-dependent $(\Sigma)$ part of the virtual-photon vertex. We find

$$
\begin{aligned}
P_{\text {recoil }}^{(11,11) S}= & P_{\text {recoil, } C}^{(11,11) S}+P_{\text {recoil }, \Sigma}^{(11,11) S}, \\
P_{\text {recoil }, C}^{(11,11) S}= & \frac{1}{18} \frac{\alpha^{2}}{m_{q}^{2}} e^{-q^{2} / 6 \alpha^{2}} \sum_{X=N^{*}, \Delta^{*}} \frac{a_{X}^{2} Z_{1111, C}^{S, J_{X}}}{E_{X}(q)\left[E(q)-E_{X}(q)\right]^{2}}\left[1-\frac{2 E_{X}(q)\left[E(q)-E_{X}(q)\right]}{3 \alpha^{2}}\right], \\
P_{\text {recoil }, \Sigma}^{(11,11) S}= & \frac{1}{18} \frac{q^{2}}{m_{q}^{2}} e^{-q^{2} / 6 \alpha^{2}} \sum_{X=N^{*}, \Delta^{*}} a_{X}^{2} \frac{(-1)^{I_{x}-1 / 2}}{2 I_{x}} \frac{Z_{1111, \Sigma}^{S, J_{X}}}{E_{X}(q)\left[E(q)-E_{X}(q)\right]^{2}} \\
& \times\left[1-\frac{E_{X}(q)\left[E(q)-E_{X}(q)\right]}{3 \alpha^{2}}\right], \\
\hat{P}_{\text {recoil }}^{(11,2) S}= & \hat{P}_{\text {recoil }, C}^{(11,2) S}+\hat{P}_{\text {recoil }, \Sigma}^{(11,2) S}, \\
\hat{P}_{\text {recoil }, C}^{(11,2) 1}= & -\frac{1}{6 \sqrt{5}} \frac{1}{m_{q}^{2}} e^{-q^{2} / 6 \alpha^{2}} \sum_{X=N^{*}, \Delta^{*}} \frac{a_{X}^{2} Z_{1102}^{J_{X}}}{E_{X}(q)\left[E(q)-E_{X}(q)\right]^{2}}\left[1-\frac{E_{X}(q)\left[E(q)-E_{X}(q)\right]}{3 \alpha^{2}}\right], \\
\hat{P}_{\text {recoil }, \Sigma}^{(11,2) 1}= & \frac{1}{2 \sqrt{5}} \frac{1}{m_{q}^{2}} e^{-q^{2} / 6 \alpha^{2}} \sum_{X=N^{*}, \Delta^{*}} a_{X}^{2} \frac{(-1)^{I_{x}-1 / 2}}{2 I_{x}} \frac{Z_{102}^{J_{X}}}{E_{X}(q)\left[E(q)-E_{X}(q)\right]^{2}} \\
& \times\left[1-\frac{E_{X}(q)\left[E(q)-E_{X}(q)\right]}{3 \alpha^{2}}\right] .
\end{aligned}
$$


The values for the angular coefficients $Z$ of the recoil contributions are given in Tab. III.

\section{Comparison with Liu, Thomas, and Guichon}

We now compare our results with those of Ref. [37]. P1 As has been stated in Sec. III.A, the spin-dependent polarizabilities differ by an overall factor of three due to the different definition of the reduced multipoles in Eq. (30). In contrast with Ref. [37], we find, at leading order, two simple relations for the angular coefficients of the direct and crossed channels [see Eqs. (57)-(63)],

$$
\begin{aligned}
Z_{c}^{S, J_{X}} & =(-1)^{S} Z_{d}^{S, J_{X}}, \\
Z_{a c}^{L, S, J_{X}} & =(-1)^{S+1} Z_{a d}^{L, S, J_{X}} .
\end{aligned}
$$

In particular, as a result of Eq. (72) combined with the relative phases between the directand crossed-channel contributions to $P_{\text {para }}^{(11,11) 1}$, the leading term of the $P^{(01,01) 1}$ and $P^{(11,11) 1}$ polarizabilities vanishes at the real-photon point. This is in agreement with the constraint by photon crossing as derived in Eq. (B3) of App. B, but in contrast to the results of Ref. [37]. In addition, in our calculation the diamagnetic contribution $P_{d i a}^{(11,11) 1}$ is only $3 / 7$ of the result of Ref. [37]. We also find different expressions for $\hat{P}^{(01,1) S}$, in particular for the relative phase of the direct- and crossed-channel contributions. Furthermore, the angular coefficients $Z_{1100}^{J_{X}}$ and $Z_{1102}^{J_{X}}$ occurring in $P_{\text {recoil }}^{(11,00) 1}$ and $P_{\text {recoil }}^{(11,02) 1}$ are smaller by a factor of $1 / 2$. In addition, we find a recoil contribution to $P_{\text {recoil }}^{(11,11) S}$ for both spin-flip and no-spin-flip transitions, while in Ref. [37] such a contribution is absent for $S=1$.

The numerical results of Eqs. (57)-(71) are shown in Figs. 1 and 2 together with the calculation of Ref. [37]. For the generalized electric polarizability

$$
\alpha(|\vec{q}|)=-\frac{e^{2}}{4 \pi} \sqrt{\frac{3}{2}} P^{(01,01) 0}(|\vec{q}|),
$$

the two results are in agreement (full line of Fig. 1). The discrepancies in $P^{(01,01) 1}, P^{(11,11) 1}$, and $P^{(01,12) 1}$ originate from the contributions of the crossed channel relative to the direct one. The different results for the generalized magnetic polarizability

$$
\beta(|\vec{q}|)=-\frac{e^{2}}{4 \pi} \sqrt{\frac{3}{8}} P^{(11,11) 0}(|\vec{q}|)
$$

are mainly due to the discrepancy in the calculation of the diamagnetic term, while the different evaluations of the recoil terms result only in small deviations. Finally, the differences in calculating the $\hat{P}^{(01,1) S}$ and $\hat{P}_{\text {recoil }}^{(11,2) 1}$ polarizabilities give rise to discrepancies of almost one order of magnitude. In particular, the $\hat{P}_{\text {recoil }}^{(11,2) 1}$ polarizability receives its main contribution from $\hat{P}_{r e c o i l, \Sigma}^{(11,2) 1}$ which has been neglected in Ref. [37.

\footnotetext{
${ }^{10}$ We stress that both calculations start from the same model and use the same parameters and approximations.
} 


\section{Comparison with other calculations and experiment}

In Figs. 3 and 4 we compare the two expansion schemes of Secs. III.B and III.C. In each graph the solid line represents the full result according to the scheme of Liu, Thomas, and Guichon. Recall that in this framework the energy denominators of the crossed channel contributions are written using relativistic kinematics. The dashed line corresponds to a consistent nonrelativistic expansion up to and including terms linear in $1 / M$. The relevant expressions can be found in App. D. The contributions of leading order in 1/M [see Eqs. (51) and (52)] are separately displayed as dotted lines.

First of all, we note that due to photon-crossing symmetry the leading contributions to both $P^{(01,01) 1}$ and $P^{(11,11) 1}$ vanish identically. The use of relativistic expressions in the energy denominators for the crossed-channel terms leads to pronounced differences between the two schemes, as soon as the leading term is vanishing or small. A striking example is given by the difference between the solid and dashed lines in $P^{(01,01) 1}$ of Fig. 3, which is entirely due to this different treatment of the crossed-channel energy denominators. Finally, at the real-photon point, the leading contributions of both expansion schemes are equal, whereas the recoil terms differ by the contribution of second-order terms in $1 / M$.

Figures 5 and 6 display our results (full lines) together with the predictions of the linear sigma model 40] (dashed lines), an effective Lagrangian model [39] (dotted lines), and heavy-baryon chiral perturbation theory [44] (dashed-dotted lines).

An unpolarized measurement can be analyzed in terms of three structure functions $P_{L L}, P_{T T}$, and $P_{L T}$ [30,35,46] which are products of the GPs and the electromagnetic Sachs form factors $G_{E}$ and $G_{M}, \square$

$$
\begin{aligned}
P_{L L}(|\vec{q}|)= & -2 \sqrt{6} M G_{E}\left(Q_{0}^{2}\right) P^{(01,01) 0}(|\vec{q}|), \\
P_{T T}(|\vec{q}|)= & \frac{3}{2} G_{M}\left(Q_{0}^{2}\right)\left\{2 \omega_{0} P^{(01,01) 1}(|\vec{q}|)+\sqrt{2}|\vec{q}|^{2}\left[\sqrt{3} \hat{P}^{(01,1) 1}(|\vec{q}|)+P^{(01,12) 1}(|\vec{q}|)\right]\right\} \\
= & 3 G_{M}\left(Q_{0}^{2}\right)|\vec{q}|^{2}\left[\sqrt{2} P^{(01,12) 1}(|\vec{q}|)-P^{(11,11) 1}(|\vec{q}|) / \omega_{0}\right], \\
P_{L T}(|\vec{q}|)= & \sqrt{\frac{3}{2}} \frac{M|\vec{q}|}{\sqrt{Q_{0}^{2}}} G_{E}\left(Q_{0}^{2}\right) P^{(11,11) 0}(|\vec{q}|) \\
& +\frac{\sqrt{3}}{2} \frac{\sqrt{Q_{0}^{2}}}{|\vec{q}|} G_{M}\left(Q_{0}^{2}\right)\left[P^{(11,00) 1}(|\vec{q}|)+\frac{|\vec{q}|^{2}}{\sqrt{2}} P^{(11,02) 1}(|\vec{q}|)\right] \\
= & \sqrt{\frac{3}{2}} \frac{M|\vec{q}|}{\sqrt{Q_{0}^{2}}} G_{E}\left(Q_{0}^{2}\right) P^{(11,11) 0}(|\vec{q}|)+\frac{3}{2} \frac{\sqrt{Q_{0}^{2}}|\vec{q}|}{\omega_{0}} G_{M}\left(Q_{0}^{2}\right) P^{(01,01) 1}(|\vec{q}|),
\end{aligned}
$$

where $\omega_{0}=\left.\omega\right|_{\omega^{\prime}=0}=M-\sqrt{M^{2}+|\vec{q}|^{2}}$ and $Q_{0}^{2}=-\left.q^{2}\right|_{\omega^{\prime}=0}=-2 M \omega_{0}$. We note that the second equations of Eqs. (75) and(76), respectively, rely on symmetry under particle crossing and charge conjugation [see Eqs. (21c) and (21d) of Ref. [35]] which is not satisfied

\footnotetext{
${ }^{11}$ The fourth structure function $P_{L T}^{\prime}$ of Ref. [30] is related to $P_{L T}$ if symmetry under particle crossing and charge conjugation is applied [35].
} 
in the NRCQM. Table IV contains the predictions for the three response functions for $|\vec{q}|=$ $(0,240,600) \mathrm{MeV}$, corresponding to the real-photon point, MIT-Bates [48, and MAMI 46] kinematics, respectively. For the Sachs form factors we used the parametrization of Ref. [58]. For $P_{T T}$ and $P_{L T}$ we quote both results obtained from Eqs. (75) and (76), respectively.

Finally, in table $\mathrm{V}$ we compare the predictions with the first experimental information obtained at MAMI [46] for the linear combination $P_{L L}-P_{T T} / \epsilon$ and $P_{L T}$ at $|\vec{q}|=600$ $\mathrm{MeV}$.

\section{E. Particle crossing, charge conjugation, and gauge invariance}

The original definition of the GPs of Ref. [30] was based on angular momentum conservation, parity conservation, and gauge invariance. In Refs. 34,35] it was shown that only six of the originally ten GPs are independent if particle-crossing symmetry in combination with charge-conjugation invariance is imposed. As a result a set of four linear equations was obtained,

$$
\begin{aligned}
& \hat{P}^{(01,1) 0}=-\frac{\omega_{0}}{|\vec{q}|^{2}}\left[\sqrt{\frac{2}{3}} P^{(01,01) 0}+\frac{1}{\sqrt{6}} P^{(11,11) 0}\right] \\
& \hat{P}^{(11,2) 1}=-\frac{1}{|\vec{q}|^{2}}\left[\sqrt{\frac{2}{5}} P^{(11,11) 1}+\sqrt{\frac{3}{5}} \omega_{0} P^{(11,02) 1}\right], \\
& \hat{P}^{(01,1) 1}=-\frac{1}{|\vec{q}|^{2}}\left[\sqrt{\frac{2}{3}} \omega_{0} P^{(01,01) 1}+\sqrt{\frac{2}{3}} \frac{|\vec{q}|^{2}}{\omega_{0}} P^{(11,11) 1}-\frac{1}{\sqrt{3}}|\vec{q}|^{2} P^{(01,12) 1}\right], \\
& P^{(11,00) 1}=\sqrt{3} \frac{|\vec{q}|^{2}}{\omega_{0}} P^{(01,01) 1}-\frac{1}{\sqrt{2}}|\vec{q}|^{2} P^{(11,02) 1},
\end{aligned}
$$

where $\omega_{0}=\left.\omega\right|_{\omega^{\prime}=0}$. Following common use, we choose for the six independent GPs $\alpha, \beta$, $P^{(01,01) 1}, P^{(11,11) 1}, P^{(01,12) 1}$, and $P^{(11,02) 1}$ as given in Figs. 1,3 , and 5 .

However, in a nonrelativistic framework particle crossing is not a symmetry of the Compton tensor (see Sec. 2.3 of Ref. [55]). Thus one cannot expect the relations of Eqs. (77) - (80) to be satisfied in the NRCQM. Having defined $\Delta_{i}(i=1,2,3,4)$ as the difference between the left-hand and right-hand sides of Eqs. (77) - (80) normalized to their right-hand sides, we show the discrepancies $\Delta_{i}$ in Fig. 7 as function of $|\vec{q}|$. Clearly, the relations of Eqs. (77) - (80) are strongly violated on the average.

Finally, another important limitation of the NRCQM is due to a violation of gauge invariance. This problem can be traced back to essentially two causes: first, the Isgur-Karl model includes some effects of the anharmonic terms in the $q q$ potential only perturbatively in the energy but not in the wave functions. Such a treatment leads to a mismatch between the resonance masses in the energy denominators of the Compton amplitude and the baryon states which enter in the current matrix elements. Second, the actual calculation truncates the configuration space to only few intermediate states, while gauge invariance requires, in principle, the full set of intermediate states. The well-known example in this context is, of course, the low-energy Thomson limit which requires the inclusion 
of the sum over all electric-dipole excitations (including the negative-energy states of a relativistic theory).

\section{SUMMARY AND CONCLUSIONS}

We discussed the general form of the virtual Compton scattering tensor for a nonrelativistic composite system. In particular, we focussed the attention on the generalized polarizabilities of the proton, defined from the multipole expansion of the Compton tensor. We performed a consistent nonrelativistic expansion of the structure-dependent amplitude allowing us to identify the constraints due to photon crossing. As a model calculation, we reconsidered the proton GPs in a nonrelativistic constituent quark model. The model satisfies the constraint due to photon crossing at the real photon point, but does not provide the relations among the GPs due to nucleon crossing in combination with charge conjugation. As a consequence of its limitations regarding relativity, gauge invariance and chiral symmetry, the results of the model should be treated with some care. There clearly is room for improvement in any of the above-mentioned shortcomings. Nonetheless, the predictions provide an order-of-magnitude estimate for the nucleon resonance contributions and as such are complementary to the results of the linear sigma model and chiral perturbation theory emphasizing pionic degrees of freedom and chiral symmetry.

\section{ACKNOWLEDGMENTS}

We would like to thank P. A. M. Guichon for useful comments on the manuscript. B.P. is grateful to A. Metz and M. Vanderhaeghen for stimulating discussions. This work was supported in part by the Deutsche Forschungsgemeinschaft (SFB 443).

\section{APPENDIX A: GAUGE-INVARIANT MODIFIED GROUNDSTATE POLE TERMS}

Here we derive the result of Eq. (25) for $G^{\mu \nu}$ which, in combination with the groundstate pole terms, constitutes the gauge-invariant tensor $\tilde{M}_{P}^{\mu \nu}$. We start with the expression [see Eqs. (8a,b) of Ref. [52] with the replacement $k \rightarrow q^{\prime}$ and $k^{\prime} \rightarrow-q$ ]

$$
T_{f i}^{\mu \nu}\left(q^{\prime}, q, \vec{p}\right)=-i(2 \pi)^{3} \int d^{4} z e^{i q^{\prime} \cdot z}\left\langle 0 M_{f} \vec{p}_{f}\left|T\left[J^{\mu}(z) J^{\nu}(0)\right]\right| 0 M_{i} \vec{p}_{i}\right\rangle,
$$

where $J^{\mu}(x)=\exp \left(i H_{0} t\right) J^{\mu}(\vec{x}) \exp \left(-i H_{0} t\right)$. In general, we allow for a change in the spin projection from $M_{i}$ to $M_{f}$. The groundstate pole contribution is given by

$$
\begin{aligned}
& T_{P, f i}^{\mu \nu}\left(q^{\prime}, q, \vec{p}\right)= \\
& \begin{aligned}
-i(2 \pi)^{3} \sum_{M} \int d^{3} P \int d^{4} z e^{i q^{\prime} \cdot z}[ & \theta\left(z_{0}\right)\left\langle 0 M_{f} \vec{p}_{f}\left|J^{\mu}(z)\right| 0 M \vec{P}\right\rangle\left\langle 0 M \vec{P}\left|J^{\nu}(0)\right| 0 M_{i} \vec{p}_{i}\right\rangle \\
& \left.+\theta\left(-z_{0}\right)\left\langle 0 M_{f} \vec{p}_{f}\left|J^{\nu}(0)\right| 0 M \vec{P}\right\rangle\left\langle 0 M \vec{P}\left|J^{\mu}(z)\right| 0 M_{i} \vec{p}_{i}\right\rangle\right] .
\end{aligned}
\end{aligned}
$$


The following procedure is very similar to the one used in deriving Ward-FradkinTakahashi identities [59 61] in Quantum Field Theory [see, e.g., Chap. 6.1 of Ref. [62]]. Let us contract Eq. (A1) with $q_{\mu}^{\prime}$ (arguments suppressed),

$$
q_{\mu}^{\prime} T_{P, f i}^{\mu \nu}=-i(2 \pi)^{3} \sum_{M} \int d^{3} P \int d^{4} z\left(-i \partial_{\mu} e^{i q^{\prime} \cdot z}\right)[\cdots] .
$$

Symbolically this expression is of the type

$$
\int d^{4} z\left(\partial_{\mu} e^{i q^{\prime} \cdot z}\right) f^{\mu}(z)=-\int d^{4} z e^{i q^{\prime} \cdot z} \partial_{\mu} f^{\mu}(z),
$$

where we made use of a partial integration, and assumed that the interaction is "adiabatically" switched on and off to get rid of the surface terms at $z_{0}= \pm \infty$. Similarly, use of the divergence theorem has been made. After applying this result to the above case,

$$
q_{\mu}^{\prime} T_{P, f i}^{\mu \nu}=(2 \pi)^{3} \sum_{M} \int d^{3} P \int d^{4} z e^{i q^{\prime} \cdot z} \partial_{\mu}[\cdots]
$$

we use the relation

$$
\partial_{\mu}\left[\theta\left( \pm z_{0}\right) g^{\mu}(z)\right]= \pm \delta\left(z_{0}\right) g^{0}(z)+\theta\left( \pm z_{0}\right) \partial_{\mu} g^{\mu}(z)
$$

and obtain

$$
\begin{aligned}
q_{\mu}^{\prime} T_{P, f i}^{\mu \nu}=(2 \pi)^{3} \sum_{M} \int d^{3} P \int d^{4} z e^{i q^{\prime} \cdot z} & {\left[\theta\left(z_{0}\right)\left\langle 0 M_{f} \vec{p}_{f}\left|\partial_{\mu} J^{\mu}(z)\right| 0 M \vec{P}\right\rangle\left\langle 0 M \vec{P}\left|J^{\nu}(0)\right| 0 M_{i} \vec{p}_{i}\right\rangle\right.} \\
& +\theta\left(-z_{0}\right)\left\langle 0 M_{f} \vec{p}_{f}\left|J^{\nu}(0)\right| 0 M \vec{P}\right\rangle\left\langle 0 M \vec{P}\left|\partial_{\mu} J^{\mu}(z)\right| 0 M_{i} \vec{p}_{i}\right\rangle \\
& +\delta\left(z_{0}\right)\left\langle 0 M_{f} \vec{p}_{f}|\rho(z)| 0 M \vec{P}\right\rangle\left\langle 0 M \vec{P}\left|J^{\nu}(0)\right| 0 M_{i} \vec{p}_{i}\right\rangle \\
& \left.-\delta\left(z_{0}\right)\left\langle 0 M_{f} \vec{p}_{f}\left|J^{\nu}(0)\right| 0 M \vec{P}\right\rangle\left\langle 0 M \vec{P}|\rho(z)| 0 M_{i} \vec{p}_{i}\right\rangle\right] .
\end{aligned}
$$

With $\partial_{\mu} J^{\mu}(z)=0$ as an operator identity, the first two terms on the right-hand side of the equation vanish. Performing the integration with respect to $z_{0}$, applying translational invariance as $\rho(\vec{z})=\exp (-i \hat{\vec{P}} \cdot \vec{z}) \rho(0) \exp (i \hat{\vec{P}} \cdot \vec{z})$, integrating first with respect to $\vec{z}$ and then with respect to $\vec{P}$, the two remaining terms yield

$$
\begin{aligned}
q_{\mu}^{\prime} T_{P, f i}^{\mu \nu}=(2 \pi)^{6} \sum_{M} & {\left[\left\langle 0 M_{f} \vec{p}_{f}|\rho(0)| 0 M \vec{p}_{f}+\vec{q}^{\prime}\right\rangle\left\langle 0 M \vec{p}_{f}+\vec{q}^{\prime}\left|J^{\nu}(0)\right| 0 M_{i} \vec{p}_{i}\right\rangle\right.} \\
& \left.-\left\langle 0 M_{f} \vec{p}_{f}\left|J^{\nu}(0)\right| 0 M \vec{p}_{i}-\vec{q}^{\prime}\right\rangle\left\langle 0 M \vec{p}_{i}-\vec{q}^{\prime}|\rho(0)| 0 M_{i} \vec{p}_{i}\right\rangle\right] .
\end{aligned}
$$

Finally, the operator $\rho$ of Eq. (8) is diagonal in the spin projections and we obtain

$$
\begin{array}{r}
q_{\mu}^{\prime} T_{P, f i}^{\mu \nu}=(2 \pi)^{6}\left[\left\langle 0 M_{f} \vec{p}_{f}|\rho(0)| 0 M_{f} \vec{p}_{f}+\vec{q}^{\prime}\right\rangle\left\langle 0 M_{f} \vec{p}_{f}+\vec{q}^{\prime}\left|J^{\nu}(0)\right| 0 M_{i} \vec{p}_{i}\right\rangle\right. \\
\left.-\left\langle 0 M_{f} \vec{p}_{f}\left|J^{\nu}(0)\right| 0 M_{i} \vec{p}_{i}-\vec{q}^{\prime}\right\rangle\left\langle 0 M_{i} \vec{p}_{i}-\vec{q}^{\prime}|\rho(0)| 0 M_{i} \vec{p}_{i}\right\rangle\right] .
\end{array}
$$

First we consider Eq. (A2) for $\nu=0$ :

$$
q_{\mu}^{\prime} T_{P, f i}^{\mu 0}=\left[\left\langle 0 M_{f}\left|\rho\left(-\vec{q}^{\prime}\right)\right| 0 M_{f}\right\rangle\left\langle 0 M_{f}|\rho(\vec{q})| 0 M_{i}\right\rangle-\left\langle 0 M_{f}|\rho(\vec{q})| 0 M_{i}\right\rangle\left\langle 0 M_{i}\left|\rho\left(-\vec{q}^{\prime}\right)\right| 0 M_{i}\right\rangle\right]=0,
$$


where we inserted Eqs. (14) and (17) and made use of the diagonal nature of $\rho$. Similarly, inserting Eqs. (14) - (17) for $\nu=j$ we obtain

$$
\begin{aligned}
q_{\mu}^{\prime} T_{P, f i}^{\mu j}= & \left\langle 0 M_{f}\left|\rho\left(-\vec{q}^{\prime}\right)\right| 0 M_{f}\right\rangle\left[\left\langle 0 M_{f}\left|j^{i n, j}(\vec{q})\right| 0 M_{i}\right\rangle+\frac{p_{f}^{j}+q^{\prime j}+p_{i}^{j}}{2 M}\left\langle 0 M_{f}|\rho(\vec{q})| 0 M_{i}\right\rangle\right] \\
& -\left[\left\langle 0 M_{f}\left|j^{i n, j}(\vec{q})\right| 0 M_{i}\right\rangle+\frac{p_{f}^{j}+p_{i}^{j}-q^{\prime j}}{2 M}\left\langle 0 M_{f}|\rho(\vec{q})| 0 M_{i}\right\rangle\right]\left\langle 0 M_{i}\left|\rho\left(-\vec{q}^{\prime}\right)\right| 0 M_{i}\right\rangle \\
= & \left\langle 0 M_{f}\left|j^{i n, j}(\vec{q})\right| 0 M_{i}\right\rangle\left[\left\langle 0 M_{f}\left|\rho\left(-\vec{q}^{\prime}\right)\right| 0 M_{f}\right\rangle-\left\langle 0 M_{i}\left|\rho\left(-\vec{q}^{\prime}\right)\right| 0 M_{i}\right\rangle\right] \\
& +\frac{q^{\prime j}}{M}\left\langle 0 M_{f}\left|\rho\left(-\vec{q}^{\prime}\right)\right| 0 M_{f}\right\rangle\left\langle 0 M_{f}|\rho(\vec{q})| 0 M_{f}\right\rangle \delta_{M_{i} M_{f}} \\
= & \frac{q^{\prime j}}{M}\left\langle 0 M_{f}\left|\rho\left(-\vec{q}^{\prime}\right)\right| 0 M_{f}\right\rangle\left\langle 0 M_{i}|\rho(\vec{q})| 0 M_{i}\right\rangle \delta_{M_{i} M_{f}},
\end{aligned}
$$

where we made use of the fact that the groundstate matrix elements of $\rho$ are diagonal and do not depend on the projection.

The calculation of $q_{\nu} T_{P, f i}^{\mu \nu}$ proceeds in a completely analogous fashion. Equations (A3) and (A4) suggest to add the term of Eq. (25),

$$
G^{\mu 0}\left(q^{\prime}, q\right)=G^{0 \nu}\left(q^{\prime}, q\right)=0, \quad G^{i j}\left(q^{\prime}, q\right)=\delta_{i j} \frac{1}{M}\left\langle 0 M_{f}\left|\rho\left(-\vec{q}^{\prime}\right)\right| 0 M_{f}\right\rangle\left\langle 0 M_{i}|\rho(\vec{q})| 0 M_{i}\right\rangle \delta_{M_{i} M_{f}},
$$

with the result that $\tilde{M}_{P}^{\mu \nu}=T_{P, f i}^{\mu \nu}+G^{\mu \nu}$ is gauge invariant. In particular, $\tilde{M}_{P}^{\mu \nu}$ depends on groundstate properties only.

\section{APPENDIX B: PHOTON CROSSING CONSTRAINTS AT LEADING ORDER} IN $1 / M$

In Sec. III.C we have seen that the leading term of the residual amplitude, in a $1 / M$ expansion, satisfies the photon crossing constraint [see Eq. (44)]

$$
T_{f i ; X, l . o .}^{\mu \nu}\left(q^{\prime}, q\right)=T_{f i ; X, l . o .}^{\nu \mu}\left(-q,-q^{\prime}\right) \text {. }
$$

Because Eq. (B1) does not depend on the average target momentum, it is also symmetric under "naive photon crossing,"

$$
T_{X, l . o .}^{\mu \nu}\left(M_{f}, \omega^{\prime}, \vec{q}^{\prime} ; M_{i}, \omega, \vec{q}\right)=T_{X, l . o .}^{\nu \mu}\left(M_{f},-\omega,-\vec{q} ; M_{i},-\omega^{\prime},-\vec{q}^{\prime}\right),
$$

which in terms of the multipole expansion of Eq. (27) implies

$$
\begin{aligned}
& 4 \pi \sum_{\substack{\rho, L, M, \rho^{\prime}, L^{\prime}, M^{\prime}}} g_{\rho^{\prime} \rho^{\prime}} V^{\mu}\left(\rho^{\prime} L^{\prime} M^{\prime}, \hat{q}^{\prime}\right) H_{X, l . o .}^{\left(\rho^{\prime} L^{\prime} M^{\prime}, \rho L M\right)}\left(M_{f}, \omega^{\prime},\left|\vec{q}^{\prime}\right| ; M_{i}, \omega,|\vec{q}|\right) V^{\nu *}(\rho L M, \hat{q}) g_{\rho \rho} \\
&= 4 \pi \sum_{\substack{\rho, L, M, \rho^{\prime}, L^{\prime}, M^{\prime}}} g_{\rho \rho} V^{\nu}(\rho L M,-\hat{q}) H_{X, l . o .}^{\left(\rho L M, \rho^{\prime} L^{\prime} M^{\prime}\right)}\left(M_{f},-\omega,|\vec{q}| ; M_{i},-\omega^{\prime},\left|\vec{q}^{\prime}\right|\right) V^{\mu *}\left(\rho^{\prime} L^{\prime} M^{\prime},-\hat{q}^{\prime}\right) g_{\rho^{\prime} \rho^{\prime}} \\
&= 4 \pi \sum_{\substack{\rho, L, M, \rho^{\prime}, L^{\prime}, M^{\prime}}}(-)^{L+M+L^{\prime}+M^{\prime}} V^{\mu}\left(\rho^{\prime} L^{\prime}-M^{\prime}, \hat{q}^{\prime}\right) H_{X, l . o .}^{\left(\rho L M, \rho^{\prime} L^{\prime} M^{\prime}\right)}\left(M_{f},-\omega,|\vec{q}| ; M_{i},-\omega^{\prime},\left|\vec{q}^{\prime}\right|\right) \\
& \quad \times V^{\nu *}(\rho L-M, \hat{q}) g_{\rho^{\prime} \rho^{\prime} .}
\end{aligned}
$$


In the last step we made use of

$$
V^{\mu *}(\rho L M,-\hat{q})=g_{\rho \rho}(-)^{L+M} V^{\mu}(\rho L-M, \hat{q}) .
$$

With the orthogonality property of the multipole basis $\left\{V^{\mu}(\rho L M, \hat{q})\right\}$ (see appendix $\mathrm{C}$ of Ref. [30]) we find

$$
\begin{aligned}
& H_{X, l . o .}^{\left(\rho^{\prime} L^{\prime} M^{\prime}, \rho L M\right)}\left(M_{f}, \omega^{\prime},\left|\vec{q}^{\prime}\right| ; M_{i}, \omega,|\vec{q}|\right)= \\
& (-)^{L-M+L^{\prime}-M^{\prime}} g_{\rho^{\prime} \rho^{\prime}} g_{\rho \rho} H_{X, l . o .}^{\left(\rho L-M, \rho^{\prime} L^{\prime}-M^{\prime}\right)}\left(M_{f},-\omega,|\vec{q}| ; M_{i},-\omega^{\prime},\left|\vec{q}^{\prime}\right|\right) .
\end{aligned}
$$

Because of the orthogonality relations of Clebsch-Gordan coefficients we finally obtain for the reduced multipoles

$$
\begin{aligned}
& H_{X, l . o .}^{\left(\rho^{\prime} L^{\prime}, \rho L\right) S}\left(M_{f}, \omega^{\prime},\left|\vec{q}^{\prime}\right| ; M_{i}, \omega,|\vec{q}|\right)= \\
& (-)^{L+L^{\prime}-S} g_{\rho^{\prime} \rho^{\prime}} g_{\rho \rho} H_{X, l . o .}^{\left(\rho L, \rho^{\prime} L^{\prime}\right) S}\left(M_{f},-\omega,|\vec{q}| ; M_{i},-\omega^{\prime},\left|\vec{q}^{\prime}\right|\right) .
\end{aligned}
$$

We stress that the above derivation holds only for those pieces that are independent of $\vec{p}$. It is only in this case that true photon crossing is equivalent to naive photon crossing in the center-of-mass frame.

\section{APPENDIX C: OVERLAP INTEGRALS OF THE CURRENT OPERATOR IN THE NRCQM}

The contribution of the SU(3) multiplets to the overlap integrals defined in Eqs. (54)(56) is given by $(q=|\vec{q}|)$

$$
\begin{aligned}
& \rho_{N(28)}(\vec{q})=-i \frac{\sqrt{8 \pi}}{3} \frac{q}{\alpha} e^{-q^{2} / 6 \alpha^{2}} \tau_{N}(-1)^{\frac{1}{2}+M_{X}} \sqrt{2 J_{X}+1}\left(\begin{array}{ccc}
1 & \frac{1}{2} & J_{X} \\
M_{X}-m_{N} & m_{N} & -M_{X}
\end{array}\right) Y_{1 M_{X}-m_{N}}^{*}(\hat{q}), \\
& \vec{P}_{N(28)}(\vec{q})=-i \frac{\sqrt{6}}{3} \frac{\alpha}{m_{q}} e^{-q^{2} / 6 \alpha^{2}} \tau_{N}(-1)^{\frac{1}{2}+M_{X}} \sqrt{2 J_{X}+1}\left(\begin{array}{ccc}
1 & \frac{1}{2} & J_{X} \\
M_{X}-m_{N} & m_{N} & -M_{X}
\end{array}\right) \vec{e}_{M_{X}-m_{N}}^{*}, \\
& \vec{\Sigma}_{N\left({ }^{2} 8\right)}(\vec{q})=i \frac{\sqrt{2 \pi}}{3} \frac{q}{\alpha} e^{-q^{2} / 6 \alpha^{2}}\left(1+4 \tau_{N}\right) \sum_{\mu}(-1)^{\frac{1}{2}+M_{X}} \sqrt{2 J_{X}+1}\left(\begin{array}{ccc}
1 & \frac{1}{2} & J_{X} \\
M_{X}-\mu & \mu & -M_{X}
\end{array}\right) \\
& \times Y_{1 M_{X}-\mu}^{*}(\hat{q})\left\langle\chi_{\mu}^{\lambda}\left|\vec{\sigma}_{3}\right| \chi_{m_{N}}^{\lambda}\right\rangle, \\
& \vec{\Sigma}_{N\left({ }^{4} 8\right)}(\vec{q})=-i \frac{\sqrt{\pi}}{6} \frac{q}{\alpha} e^{-q^{2} / 6 \alpha^{2}}\left(1-2 \tau_{N}\right) \sum_{\mu}(-1)^{-\frac{1}{2}+M_{X}} \sqrt{2 J_{X}+1}\left(\begin{array}{ccc}
1 & \frac{3}{2} & J_{X} \\
M_{X}-\mu & \mu & -M_{X}
\end{array}\right) \\
& \times Y_{1 M_{X}-\mu}^{*}(\hat{q})\left\langle\chi_{\mu}^{\frac{3}{2}}\left|\vec{\sigma}_{3}\right| \chi_{m_{N}}^{\lambda}\right\rangle, \\
& \vec{\Sigma}_{\Delta}(\vec{q})=e^{-q^{2} / 6 \alpha^{2}}\left\langle\chi_{m_{\Delta}}^{\frac{3}{2}}\left|\vec{\sigma}_{3}\right| \chi_{m_{N}}^{\lambda}\right\rangle \\
& \rho_{\Delta\left({ }^{2} 10\right)}(\vec{q})=\frac{1}{2 \tau_{N}} \rho_{N(28)}(\vec{q}) \text {, }
\end{aligned}
$$




$$
\begin{aligned}
\vec{P}_{\Delta(210)}(\vec{q}) & =\frac{1}{2 \tau_{N}} \vec{P}_{N\left({ }^{2} 8\right)}(\vec{q}), \\
\vec{\Sigma}_{\Delta(210)}(\vec{q}) & =\frac{-1}{1+4 \tau_{N}} \vec{\Sigma}_{N(28)}(\vec{q}),
\end{aligned}
$$

where $\vec{e}_{m}$ is the spherical basis vector and $\alpha$ the oscillator parameter. The eigenstates of the total spin $S$ of the three quarks have been denoted by $\chi_{\mu}^{3 / 2}$ for $S=3 / 2$, and $\chi_{\sigma_{N}}^{\lambda}$ for $S=1 / 2$, with $\lambda$ indicating symmetry under interchange of the (12) quark pair. We note that the definitions for the overlap integrals introduced in Eqs. (54)-(56) differ by a factor 3 from the corresponding expressions in Eqs. (9)-(11) of Ref. [37. In addition, we found a different result in the explicit calculation of the integral entering into $\vec{\Sigma}_{N(28)}(\vec{q})$ (the integral in Eq. (14) of Ref. [37] is smaller than our result by a factor $2 \sqrt{2}$ ), while we agree with the results for the integrals contributing to the remaining terms given in Eqs. (12) and (13) and Eqs. (15)-(19) of Ref. [37].

\section{APPENDIX D: POLARIZABILITIES IN THE NONRELATIVISTIC $1 / M$ EXPANSION}

In this appendix we collect the results for the polarizabilities obtained from the multipole expansion of the Compton tensor in the $1 / M$ nonrelativistic limit.

The leading contributions corresponding to the terms in Eq. (51) read $(q=|\vec{q}|)$

$$
\begin{aligned}
P^{(01,01) S} & =\frac{1}{18} \frac{1}{\alpha^{2}} e^{-q^{2} / 6 \alpha^{2}} \sum_{X=N^{*}, \Delta^{*}} \frac{a_{X}^{2}}{M-M_{X}} Z_{d}^{S, J_{X}}\left(1+(-1)^{S}\right), \\
P^{(01,12) 1} & =\frac{1}{18} \sqrt{\frac{3}{5}} \frac{1}{m_{q} \alpha^{2}} e^{-q^{2} / 6 \alpha^{2}} \sum_{X=N^{*}, \Delta^{*}} a_{X}^{2} \frac{(-1)^{I_{x}-1 / 2}}{2 I_{x}} \frac{Z_{a d}^{2, S, J_{X}}}{M-M_{X}}, \\
P_{\text {para }}^{(11,11) S} & =\frac{4}{27} \frac{1}{m_{q}^{2}} e^{-q^{2} / 6 \alpha^{2}} \frac{Z_{\Delta}^{S}}{M-M_{\Delta}}\left(1+(-1)^{S}\right), \\
P_{\text {dia }}^{(11,11) S} & =\delta_{S 0} \frac{1}{3 \sqrt{6}} \frac{1}{m_{q} \alpha^{2}} e^{-q^{2} / 6 \alpha^{2}}, \\
\hat{P}_{\Sigma}^{(01,1) S} & =-\frac{1}{36 \sqrt{3}} \frac{1}{m_{q} \alpha^{2}} e^{-q^{2} / 6 \alpha^{2}} \sum_{X=N^{*}, \Delta^{*}} a_{X}^{2} \frac{(-1)^{I_{x}-1 / 2}}{2 I_{x}} \frac{Z_{a d}^{1, S, J_{X}}}{M-M_{X}}\left(1-(-1)^{S}\right) .
\end{aligned}
$$

Comparing these results with Eqs. (57)-(62) for the leading contributions of the polarizabilities in the convention of Ref. [37], we notice that $P^{(01,01) S}, P_{\text {para }}^{(11,11) S}$, and $P^{(01,12) 1}$ involve the same matrix elements of the current in both expansion schemes, while for the propagator in the crossed channel we now have $\left(M-M_{X}\right)^{-1}$ instead of $\left[E(\vec{q})-E_{X}\right]^{-1}$.

The diamagnetic contribution to $P^{(11,11) 0}$ from the two-photon interaction does not change in the $1 / M$ expansion.

The polarizability $\hat{P}^{(01,1) S}$ has a leading contribution only from the spin-dependent term of the current, $\hat{P}_{\Sigma}^{(01,1) S}$, while the contribution from the convective current corresponds to $1 / M$ corrections that are taken into account in the recoil term.

The recoil contributions can be obtained from the respective terms of Eq. (52), 


$$
\begin{aligned}
& \hat{P}_{\text {recoil }}^{(01,1) S}=\frac{\sqrt{2}}{108 \sqrt{3}} \frac{1}{m_{q} \alpha^{2}} e^{-q^{2} / 6 \alpha^{2}} \sum_{X=N^{*}, \Delta^{*}} a_{X}^{2} \frac{Z_{d}^{S, J_{X}}}{M-M_{X}}\left(1+(-1)^{S}\right), \\
& P_{\text {recoil }}^{(11,00) 1}=-\frac{1}{\sqrt{3}} \frac{q^{2}}{m_{q}} e^{-q^{2} / 6 \alpha^{2}} \sum_{X=N^{*}, \Delta^{*}} \frac{a_{X}^{2} Z_{1100}^{J_{X}}}{M\left[M-M_{X}\right]^{2}}\left[1-\frac{M\left[M-M_{X}\right]}{3 \alpha^{2}}\right], \\
& P_{\text {recoil }}^{(11,02) 1}=-\frac{1}{\sqrt{3}} \frac{1}{m_{q}} e^{-q^{2} / 6 \alpha^{2}} \sum_{X=N^{*}, \Delta^{*}} \frac{a_{X}^{2} Z_{1102}^{J_{X}}}{M\left[M-M_{X}\right]^{2}}\left[1-\frac{M\left[M-M_{X}\right]}{3 \alpha^{2}}\right], \\
& P_{\text {recoil }, C}^{(11,1) S}=\frac{1}{18} \frac{\alpha^{2}}{m_{q}^{2}} e^{-q^{2} / 6 \alpha^{2}} \sum_{X=N^{*}, \Delta^{*}} \frac{a_{X}^{2} Z_{1111, F}^{S, J_{X}}}{M\left[M-M_{X}\right]^{2}}\left[1-\frac{2 M\left[M-M_{X}\right]}{3 \alpha^{2}}\right], \\
& P_{\text {recoil }, \Sigma}^{(11,1) S}=\frac{1}{18} \frac{q^{2}}{m_{q}^{2}} e^{-q^{2} / 6 \alpha^{2}} \sum_{X=N^{*}, \Delta^{*}} a_{X}^{2} \frac{(-1)^{I_{x}-1 / 2}}{2 I_{x}} \frac{Z_{1111, \Sigma}^{S, J_{X}}}{M\left[M-M_{X}\right]^{2}} \\
& \times\left[1-\frac{M\left[M-M_{X}\right]}{3 \alpha^{2}}\right] \text {, } \\
& \hat{P}_{\text {recoil }, C}^{(11,2) 1}=-\frac{1}{6 \sqrt{5}} \frac{1}{m_{q}^{2}} e^{-q^{2} / 6 \alpha^{2}} \sum_{X=N^{*}, \Delta^{*}} \frac{a_{X}^{2} Z_{1102}^{J_{X}}}{M\left[M-M_{X}\right]^{2}}, \\
& \hat{P}_{\text {recoil }, \Sigma}^{(11,2) 1}=\frac{1}{2 \sqrt{5}} \frac{1}{m_{q}^{2}} e^{-q^{2} / 6 \alpha^{2}} \sum_{X=N^{*}, \Delta^{*}} a_{X}^{2} \frac{(-1)^{I_{x}-1 / 2}}{2 I_{x}} \frac{Z_{1102}^{J_{X}}}{M\left[M-M_{X}\right]^{2}} \\
& \times\left[1-\frac{M\left[M-M_{X}\right]}{3 \alpha^{2}}\right] \text {. }
\end{aligned}
$$

Comparing with the results in Eqs. (64)-(71), we find the same expressions for $P^{(11,00) 1}$, $P^{(11,02) 1}$, and $P^{(11,11) 1}$ after the substitutions $\left[E(q)-E_{X}(q)\right]^{-1} \rightarrow\left[M-M_{X}\right]^{-1}$ and $E_{X}^{-1}(q)\left[E(q)-E_{X}(q)\right]^{-2} \rightarrow M^{-1}\left[M-M_{X}\right]^{-2}$.

As noticed previously, the additional recoil term for $\hat{P}^{(01,1) 1}$ corresponds to the $\hat{P}_{F}^{(01,1) 1}$ term in Eq. (62). Furthermore, $\hat{P}_{\text {recoil,C }}^{(11,2) 1}$ does not contain the contribution from the convective current at the virtual-photon vertex resulting from the term proportional to $J_{c, f X}^{\nu}(\vec{q}) \delta J_{c, X i}^{\mu}\left(\vec{q}^{\prime}\right)$ in Eq. (42), because this contribution corresponds to a higher-order correction in $1 / M$. 


\section{REFERENCES}

[1] A. Klein, Phys. Rev. 99, 998 (1955).

[2] W. Thirring, Phil. Mag. 41, 1193 (1950).

[3] F. E. Low, Phys. Rev. 96, 1428 (1954).

[4] M. Gell-Mann and M. L. Goldberger, Phys. Rev. 96, 1433 (1954).

[5] B. R. Holstein, Comments Nucl. Part. Phys. 19, 221 (1990).

[6] F. J. Federspiel et. al., Phys. Rev. Lett. 67, 1511 (1991).

[7] A. Zieger et al., Phys. Lett. B 278, 34 (1992).

[8] E. L. Hallin et al., Phys. Rev. C 48, 1497 (1993).

[9] B. E. MacGibbon et al., Phys. Rev. C 52, 2097 (1995).

[10] A. L'vov, V.A. Petrun'kin, and M. Schumacher, Phys. Rev. C 55, 359 (1997).

[11] A. M. Baldin, Nucl. Phys. 18, 318 (1960).

[12] M. Damashek and F. J. Gilman, Phys. Rev. D 1, 1319 (1970).

[13] D. Babusci, G. Giordano, and G. Matone, Phys. Rev. C 57, 291 (1998).

[14] M. I. Levchuk and A. I. L'vov, Nucl. Phys. A674, 449 (2000).

[15] V. Olmos de León, PhD. Thesis, Mainz (2000).

[16] J. Schmiedmayer, H. Rauch, and P. Riehs, Phys. Rev. Lett. 61, 1065 (1988).

[17] L. Koester, W. Waschkowski, and J. Meier, Z. Phys. A 329, 229 (1988).

[18] J. Schmiedmayer, P. Riehs, J. A. Harvey, and N. W. Hill, Phys. Rev. Lett. 66, 1015 (1991).

[19] L. Koester et al., Phys. Rev. C 51, 3363 (1995).

[20] K. W. Rose et al., Phys. Lett. B 234, 460 (1990).

[21] K. W. Rose et al., Nucl. Phys. A514, 621 (1990).

[22] N. R. Kolb et al., nucl-ex/0003002.

[23] D. L. Hornidge et al., Phys. Rev. Lett. 84, 2334 (2000).

[24] S. R. Choudhury and D. Z. Freedman, Phys. Rev. 168, 1739 (1969).

[25] K. Y. Lin, Nuovo Cim. A 2, 695 (1971).

[26] S. Ragusa, Phys. Rev. D 47, 3757 (1993); 49, 3157 (1994).

[27] D. Babusci, G. Giordano, A. I. L'vov, G. Matone, and A. M. Nathan, Phys. Rev. C 58, 1013 (1998).

[28] H. W. Fearing and S. Scherer, Few-Body Syst. 23, 111 (1998).

[29] H. Arenhövel and D. Drechsel, Nucl. Phys. A233, 153 (1974).

[30] P. A. M. Guichon, G. Q. Liu, and A. W. Thomas, Nucl. Phys. A591, 606 (1995).

[31] P. A. M. Guichon and M. Vanderhaeghen, Prog. Part. Nucl. Phys. 41, 125 (1998).

[32] R. A. Berg and C. N. Lindner, Nucl. Phys. 26, 259 (1961).

[33] S. Scherer, A. Yu. Korchin, and J. H. Koch, Phys. Rev. C 54, 904 (1996).

[34] D. Drechsel, G. Knöchlein, A. Metz, and S. Scherer, Phys. Rev. C 55, 424 (1997).

[35] D. Drechsel, G. Knöchlein, A. Yu. Korchin, A. Metz, and S. Scherer, Phys. Rev. C 57, 941 (1998).

[36] D. Drechsel, G. Knöchlein, A. Yu. Korchin, A. Metz, and S. Scherer, Phys. Rev. C 58, 1751 (1998).

[37] G. Q. Liu , A. W. Thomas, and P. A. M. Guichon, Austral. J. Phys. 49, 905 (1996).

[38] M. Vanderhaeghen, Phys. Lett. B 368, 13 (1996).

[39] A. Yu. Korchin and O. Scholten, Phys. Rev. C 58, 1098 (1998).

[40] A. Metz and D. Drechsel, Z. Phys. A 356, 351 (1996); Z. Phys. A 359, 165 (1997). 
[41] T. R. Hemmert, B. R. Holstein, G. Knöchlein, and S. Scherer, Phys. Rev. D 55, 2630 (1997); Phys. Rev. Lett. 79, 22 (1997).

[42] M. Kim and D.-P. Min, Seoul National University Report No. SNUTP-97-046, hepph/9704381, 1997.

[43] B. Pasquini and G. Salmè, Phys. Rev. C 57, 2589 (1998).

[44] T. R. Hemmert, B. R. Holstein, G. Knöchlein, and D. Drechsel, Phys. Rev. D 62, $014013(2000)$.

[45] J. F. J. van den Brand et al., Phys. Rev. D 52, 4868 (1995).

[46] VCS Collaboration and A1 Collaboration (J. Roche et al.), Phys. Rev. Lett. 85, 708 (2000).

[47] G. Audit et al., CEBAF Report No. PR 93-050, 1993, http://www.jlab.org/ luminita/vcs.htm].

[48] J. Shaw et al., MIT-Bates proposal No. 97-03, 1997.

[49] V. A. Petrunkin, Nucl. Phys. 55, 197 (1964).

[50] T. E. O. Ericson and J. Hüfner, Nucl. Phys. B57, 604 (1973).

[51] J. L. Friar and M. Rosen, Ann. Phys. (N.Y.) 87, 289 (1974).

[52] J. L. Friar, Ann. Phys. (N.Y.) 95, 170 (1975).

[53] H. Arenhövel, NATO Advanced Study Institute on New Vistas in Electro-Nuclear Physics, 1985, Banff, Alberta (Plenum Press, New York, 1986).

[54] A. I. L'vov, Int. J. Mod. Phys. A 8, 5267 (1993).

[55] S. Scherer, Czech J. Phys. 49, 1307 (1999).

[56] P. A. M. Guichon, private communication.

[57] N. Isgur and G. Karl, Phys. Rev. D 18, 4187 (1978).

[58] G. Höhler, E. Pietarinen, I. Sabba-Stefanescu, F. Borkowski, G. G. Simon, V. H. Walther, and R. D. Wendling, Nucl. Phys. B114, 505 (1976).

[59] J. C. Ward, Phys. Rev. 78, 182 (1950).

[60] E. S. Fradkin, Sov. Phys. JETP 2, 361 (1956).

[61] Y. Takahashi, Nuovo Cim. 6, 371 (1957).

[62] T.-P. Cheng and L.-F. Li, Gauge Theory of Elementary Particle Physics (Oxford University Press, Oxford, 1984). 


\section{TABLES}

TABLE I. Mixing parameters for the ${ }^{2} \underline{8}$ and ${ }^{2} \underline{10}$ representation of the $\mathrm{P}$-wave baryon resonances.

\begin{tabular}{|c|c|c|c|c|c|c|}
\hline \hline$X$ & $N\left(\frac{1}{2}^{-}, 1535\right)$ & $N\left(\frac{1}{2}^{-}, 1650\right)$ & $N\left(\frac{3}{2}^{-}, 1520\right)$ & $N\left(\frac{3}{2}^{-}, 1700\right)$ & $\Delta\left(\frac{1}{2}^{-}, 1620\right)$ & $\Delta\left(\frac{3}{2}^{-}, 1700\right)$ \\
\hline$a_{X}$ & 0.85 & 0.53 & 0.99 & 0.11 & 1.0 & 1.0 \\
\hline \hline
\end{tabular}

TABLE II. Angular coefficients for the leading contribution to the GPs.

\begin{tabular}{|c|c|c|c|c|c|c|c|}
\hline$L$ & $S$ & $J_{X}$ & $Z_{d}^{S, J_{X}}$ & $Z_{c}^{S, J_{X}}$ & $Z_{a d}^{L, S, J_{X}}$ & $Z_{a c}^{L, S, J_{X}}$ & $Z_{\Delta}^{S}$ \\
\hline 1 & 0 & $1 / 2$ & $\sqrt{2 / 3}$ & $\sqrt{2 / 3}$ & $-2 / \sqrt{3}$ & $2 / \sqrt{3}$ & - \\
\hline 1 & 0 & $3 / 2$ & $2 \sqrt{2 / 3}$ & $2 \sqrt{2 / 3}$ & $2 / \sqrt{3}$ & $-2 / \sqrt{3}$ & $\sqrt{6}$ \\
\hline 1 & 1 & $1 / 2$ & $2 / 3$ & $-2 / 3$ & $-2 \sqrt{2} / 3$ & $-2 \sqrt{2} / 3$ & - \\
\hline 1 & 1 & $3 / 2$ & $-2 / 3$ & $2 / 3$ & $-\sqrt{2} / 3$ & $-\sqrt{2} / 3$ & $\overline{-1}$ \\
\hline 2 & 1 & $3 / 2$ & - & - & $\sqrt{30} / 3$ & $\sqrt{30} / 3$ & - \\
\hline
\end{tabular}

TABLE III. Angular coefficients for the recoil contribution to the GPs.

\begin{tabular}{|c|c|c|c|c|c|}
\hline$S$ & $J_{X}$ & $Z_{1100}^{J_{X}}$ & $Z_{1102}^{J_{X}}$ & $\begin{array}{l}Z_{111, C}^{S, J_{X}} \\
\end{array}$ & $\begin{array}{c}Z_{1111, \Sigma}^{S, J_{X}} \\
\end{array}$ \\
\hline 0 & $1 / 2$ & - & - & $\sqrt{\frac{2}{3}}$ & $1 \sqrt{6}$ \\
\hline 0 & $3 / 2$ & - & - & $2 \sqrt{\frac{2}{3}}$ & $-1 \sqrt{6}$ \\
\hline 1 & $1 / 2$ & $1 / 27$ & $1 / 27 \sqrt{2}$ & $-1 / 3$ & $-1 / 6$ \\
\hline 1 & $3 / 2$ & $-1 / 27$ & $-1 / 27 \sqrt{2}$ & $1 / 3$ & $1 / 6$ \\
\hline
\end{tabular}

TABLE IV. Structure functions $P_{L L}, P_{T T}$, and $P_{L T}$ in $\mathrm{GeV}^{-2}$. The two entries for $P_{T T}$ and $P_{L T}$ originate from the first and second equations of Eqs. (75) and (76), respectively.

\begin{tabular}{|c|r|r|r|r|r|}
\hline \hline & $P_{L L}$ & \multicolumn{2}{|c|}{$P_{T T}$} & \multicolumn{2}{c|}{$P_{L T}$} \\
\hline$|\vec{q}|=0 \mathrm{MeV}$ & 37.0 & -0.1 & 0.0 & -11.2 & -15.8 \\
\hline$|\vec{q}|=240 \mathrm{MeV}$ & 28.7 & -2.8 & -1.4 & -8.8 & -12.4 \\
\hline$|\vec{q}|=600 \mathrm{MeV}$ & 9.9 & -5.8 & -3.1 & -3.2 & -4.5 \\
\hline \hline
\end{tabular}


TABLE V. Structure functions $P_{L L}-P_{T T} / \epsilon$ and $P_{L T}$ for $|\vec{q}|=600 \mathrm{MeV}$ in $\mathrm{GeV}^{-2}(\epsilon=0.62)$. The two entries for $P_{L L}-P_{T T} / \epsilon$ and $P_{L T}$ in our calculation originate from the first and second equation of Eqs. (75) and (76), respectively.

\begin{tabular}{|l|r|c|c|c|}
\hline \hline & \multicolumn{2}{|c|}{$P_{L L}-P_{T T} / \epsilon$} & \multicolumn{2}{c|}{$P_{L T}$} \\
\hline This calculation & 19.2 & 14.9 & -3.2 & -4.5 \\
\hline NRCQM of [37] & \multicolumn{2}{|c|}{11.1} & \multicolumn{2}{c|}{-3.5} \\
\hline Experiment [46] & \multicolumn{2}{|c|}{23.7} & \multicolumn{2}{c|}{-5.0} \\
& $\pm 2.2 \pm 0.6 \pm 4.3$ & $\pm 0.8 \pm 1.1 \pm 1.4$ \\
\hline \hline
\end{tabular}




\section{FIGURES}
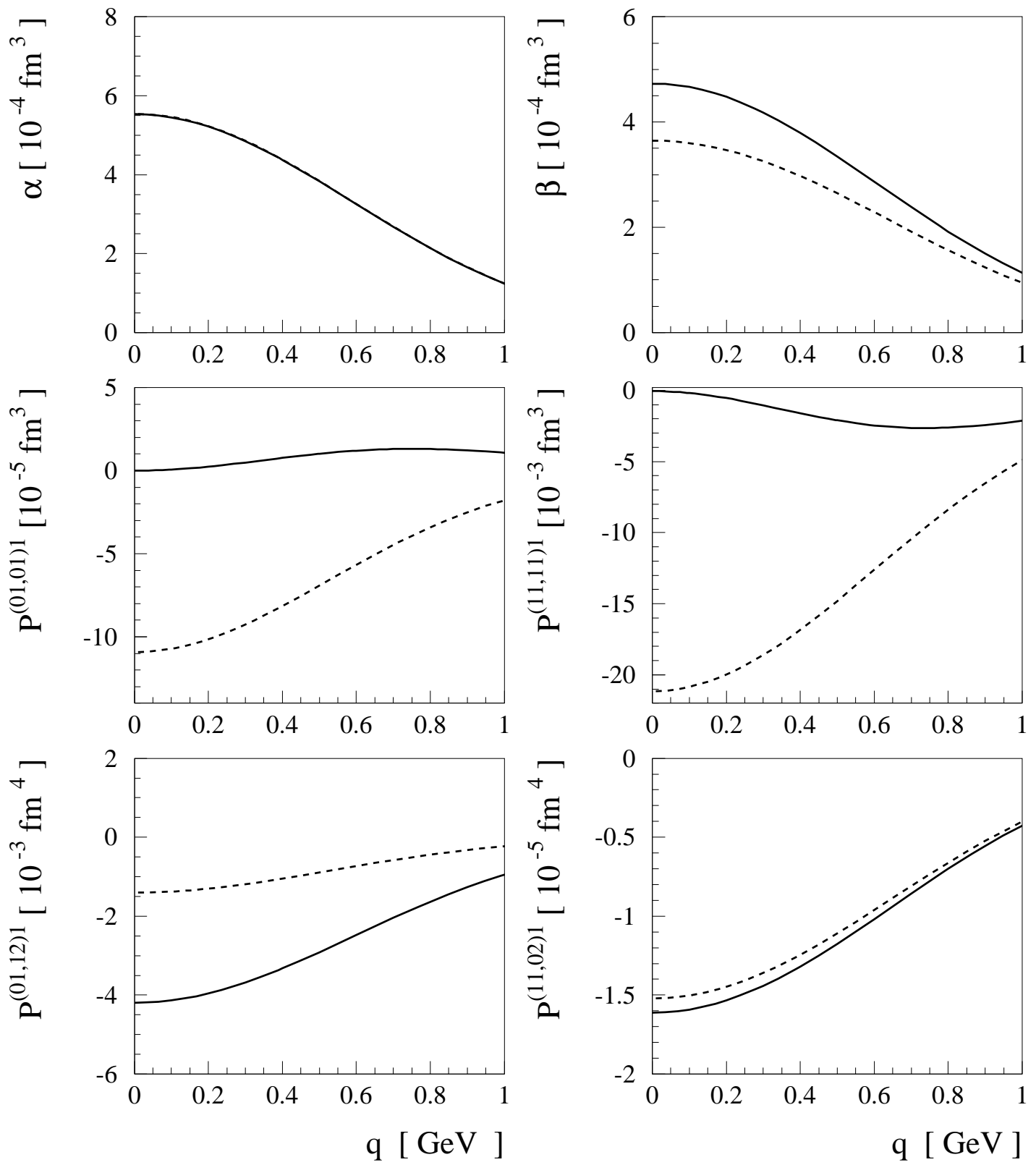

FIG. 1. GPs in the NRCQM as function of virtual-photon momentum $q=|\vec{q}|$. Full lines: our results in the scheme of LTG, taking account of leading and recoil terms; dashed lines: calculation of Ref. [37]. Note that the two calculations coincide in the case of $\alpha$. 

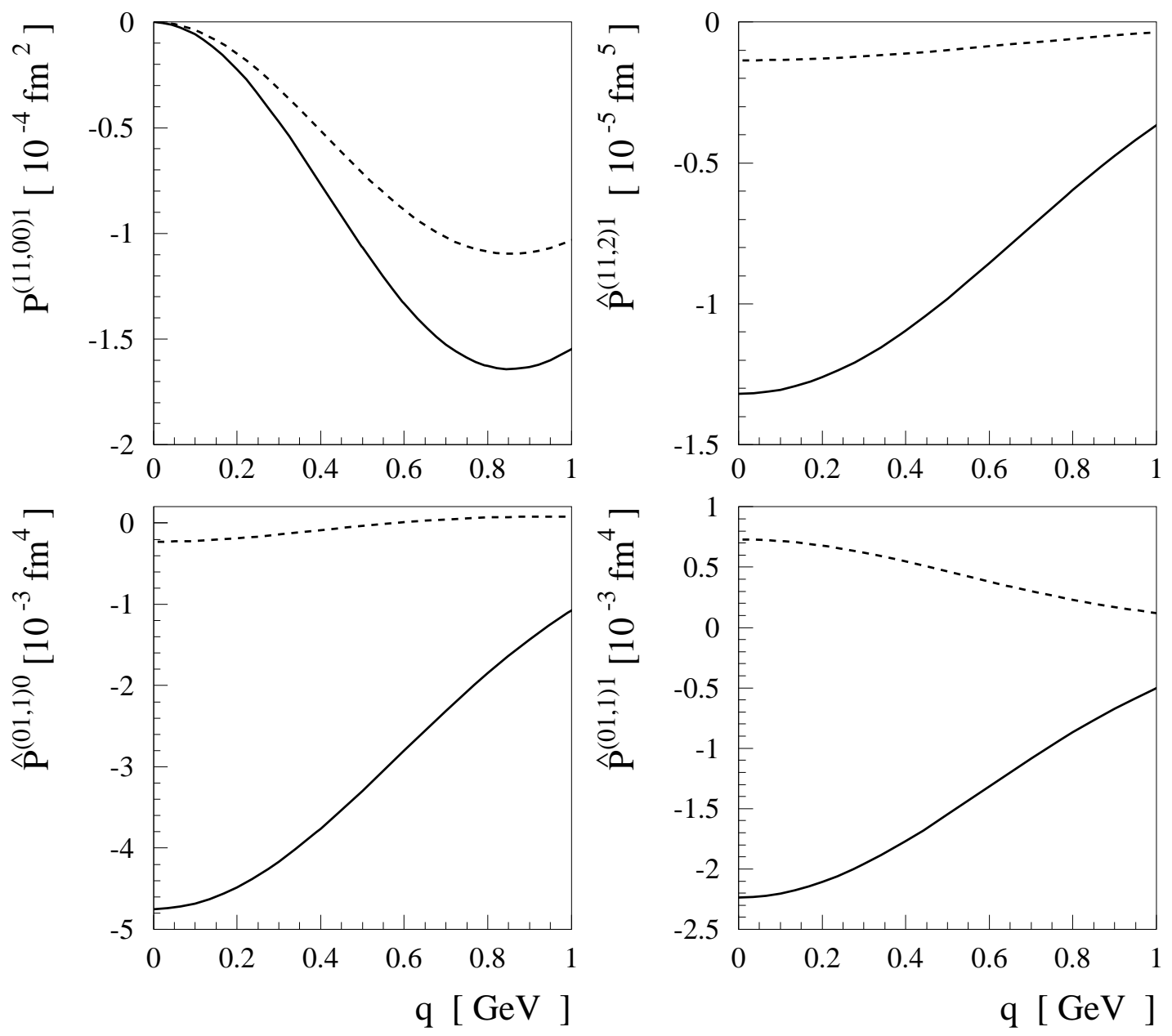

FIG. 2. GPs in the NRCQM as function of virtual-photon momentum $q=|\vec{q}|$. Full lines: our results in the scheme of LTG, taking account of leading and recoil terms; dashed lines: calculation of Ref. 37. 

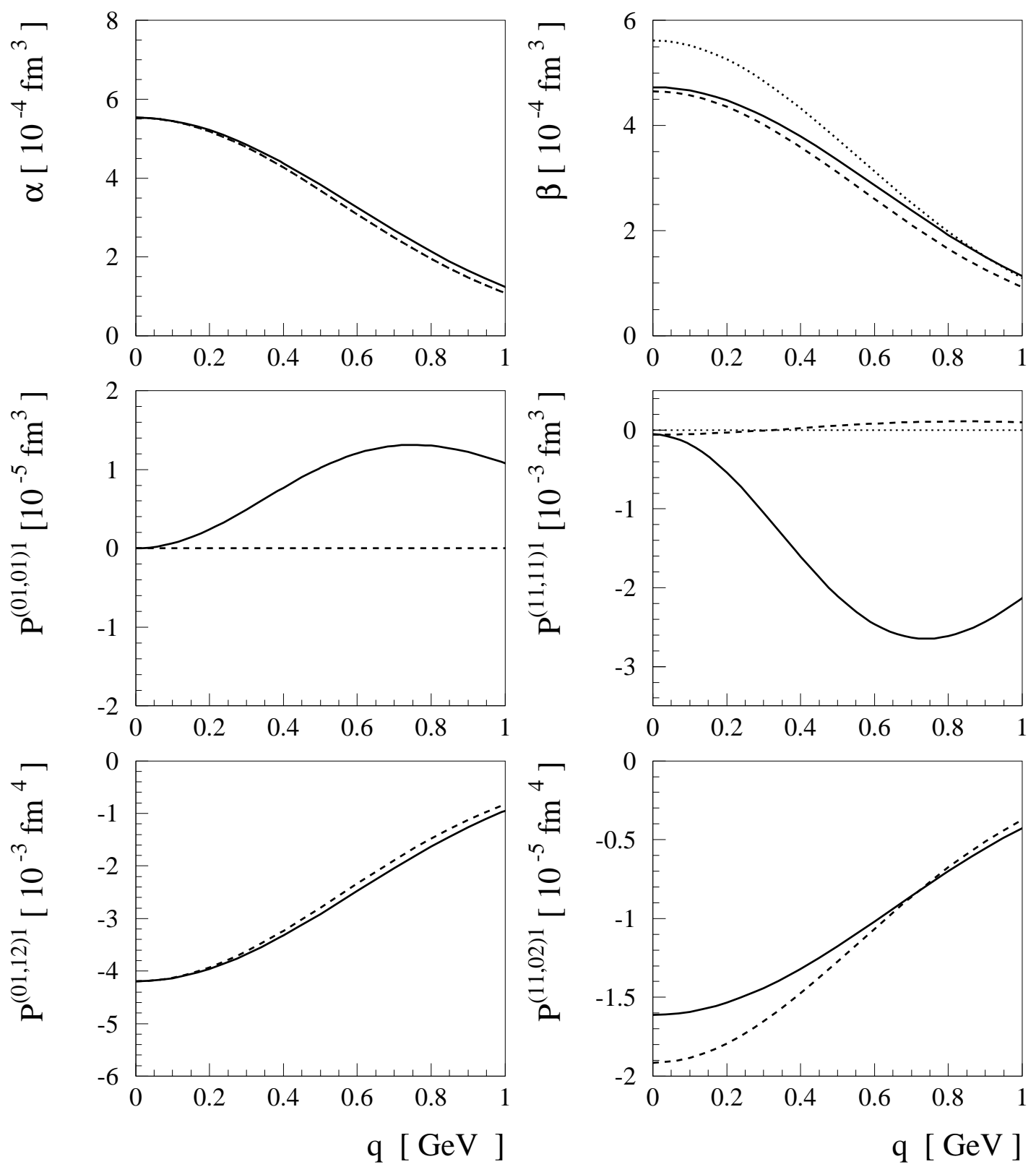

FIG. 3. GPs in the NRCQM as function of virtual-photon momentum $q=|\vec{q}|$. Full lines: our results in the scheme of LTG, including the leading and recoil contributions; dashed lines: nonrelativistic $1 / M$ expansion of the Compton tensor, obtained from the sum of the leading and recoil terms; dotted lines: leading contributions. Note that dotted lines are only included if the leading contribution is finite and different from the total result. To the order considered, the polarizabilities $\alpha, P^{(01,12) 1}$, and $P^{(01,01) 1}$ receive only contributions from the leading terms of Eq. (51), whereas $P^{(11,02) 1}$ consists only of a recoil contribution from Eq. (52). 

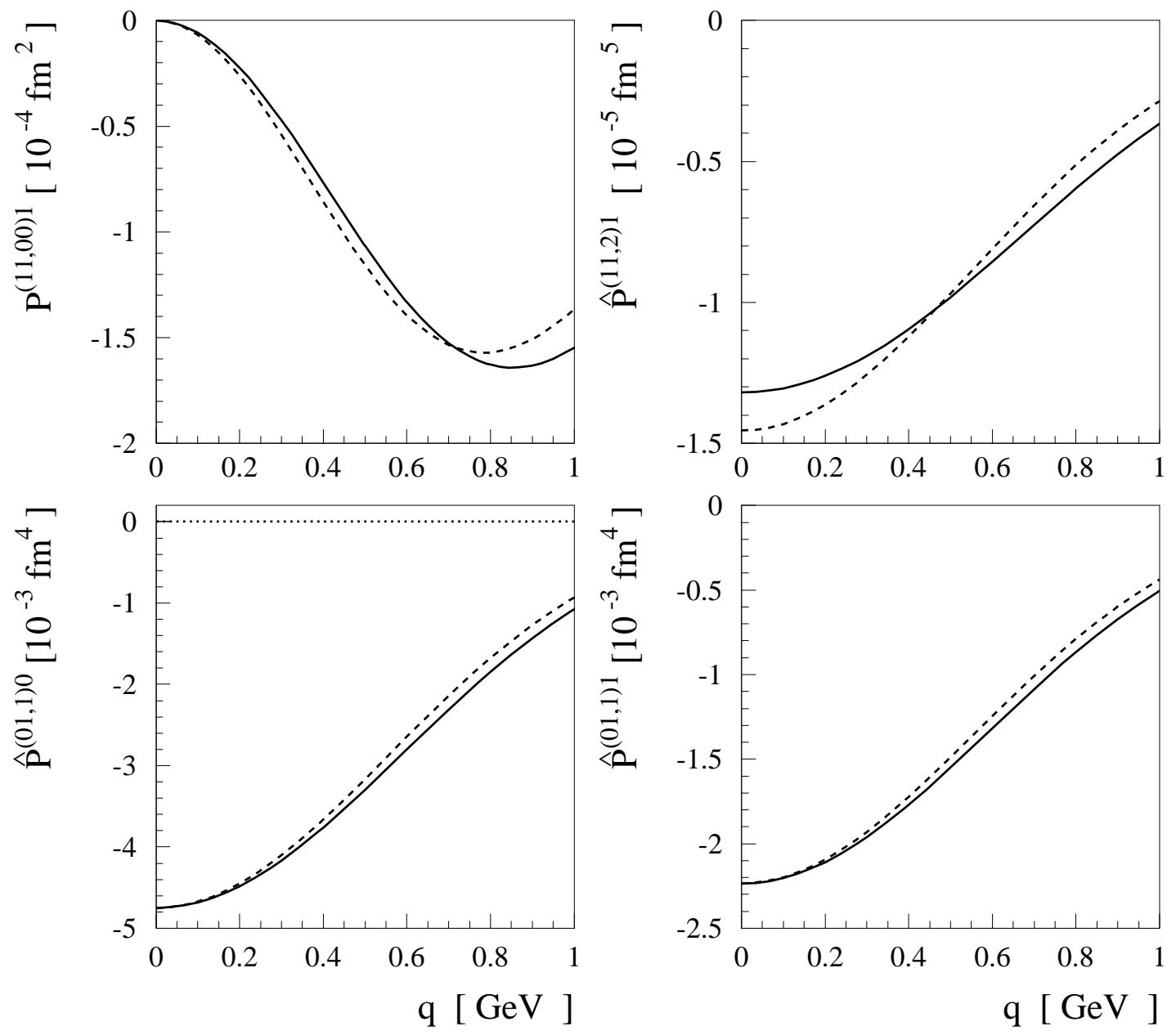

FIG. 4. GPs in the NRCQM as function of virtual-photon momentum $q=|\vec{q}|$. Full lines: our results in the scheme of LTG, including the leading and recoil contribution; dashed lines: nonrelativistic $1 / M$ expansion of the Compton tensor, obtained from the sum of leading and recoil terms; dotted lines: leading contributions. Note that dotted lines are only included if the leading contribution is finite and different from the total result. To the order considered, $\hat{P}^{(01,1) 1}$ receives only a contribution from the leading terms of Eq. (51), whereas $P^{(11,00) 1}$ and $\hat{P}^{(11,2) 1}$ consist only of a recoil contribution from Eq. (52). 

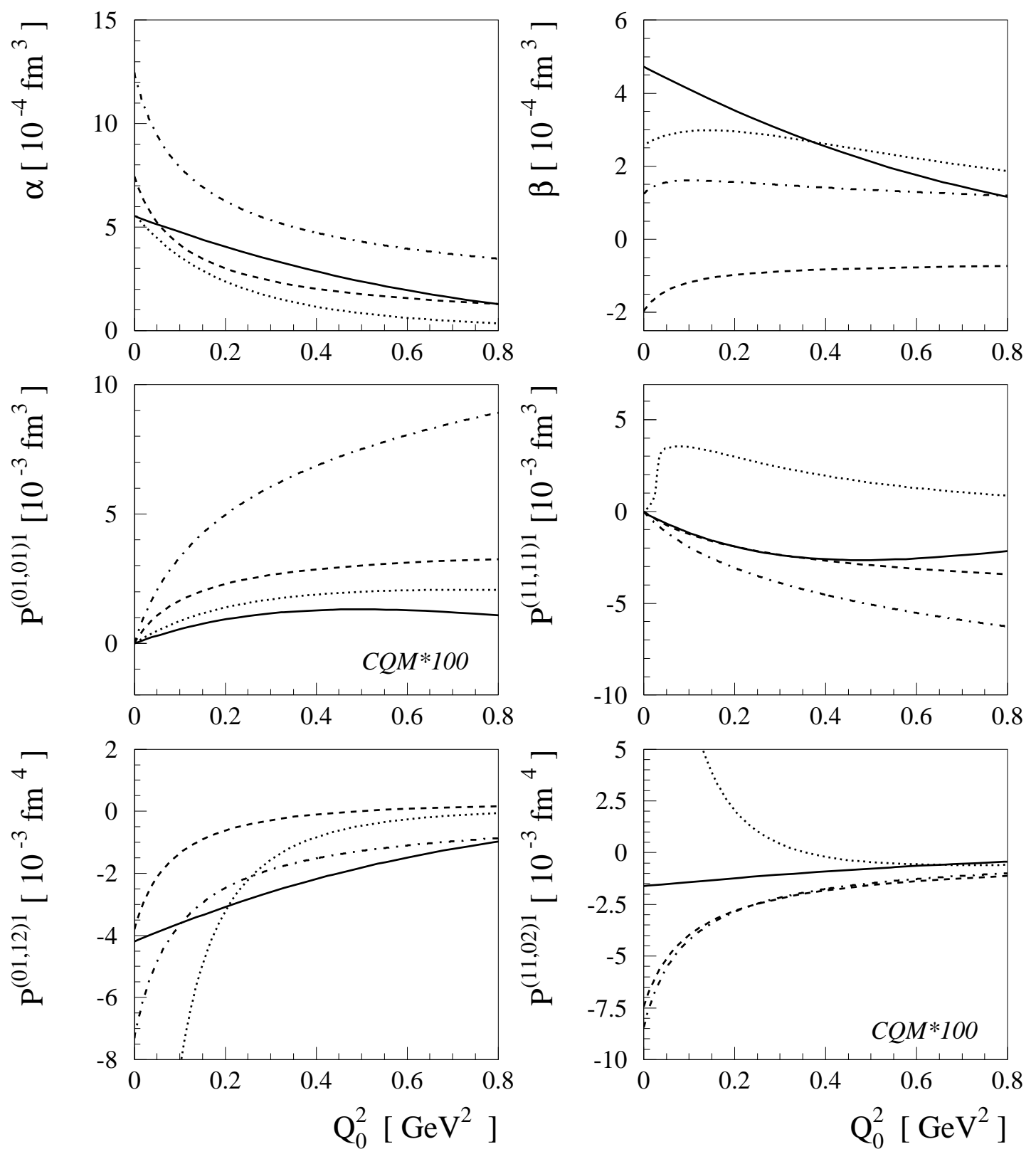

FIG. 5. Results for GPs in different model calculations as function of squared momentum transfer at $\omega^{\prime}=0, Q_{0}^{2}=\left.Q^{2}\right|_{\omega^{\prime}=0}$. Full lines: our results in the NRCQM with the scheme of LTG; dashed lines: linear sigma model [40]; dotted lines: effective Lagrangian model [39]; dashed-dotted lines : heavy-baryon chiral perturbation theory 44]. 

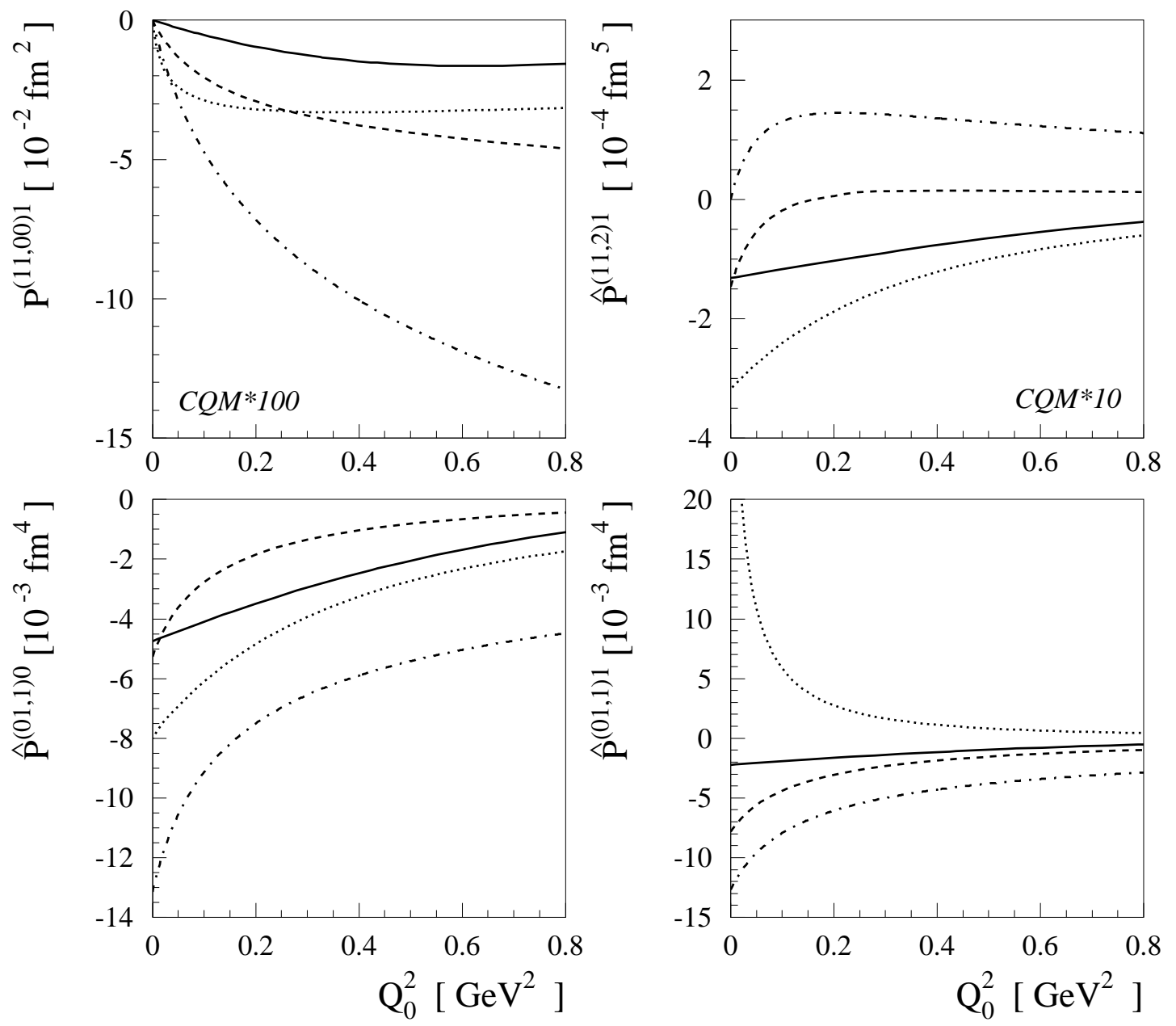

FIG. 6. Results for GPs in different model calculations as function of squared momentum transfer at $\omega^{\prime}=0, Q_{0}^{2}=\left.Q^{2}\right|_{\omega^{\prime}=0}$. Full lines: our results in the NRCQM with the scheme of LTG; dashed lines: linear sigma model [40]; dotted lines: effective Lagrangian model [39]; dashed-dotted lines : heavy-baryon chiral perturbation theory 44. 

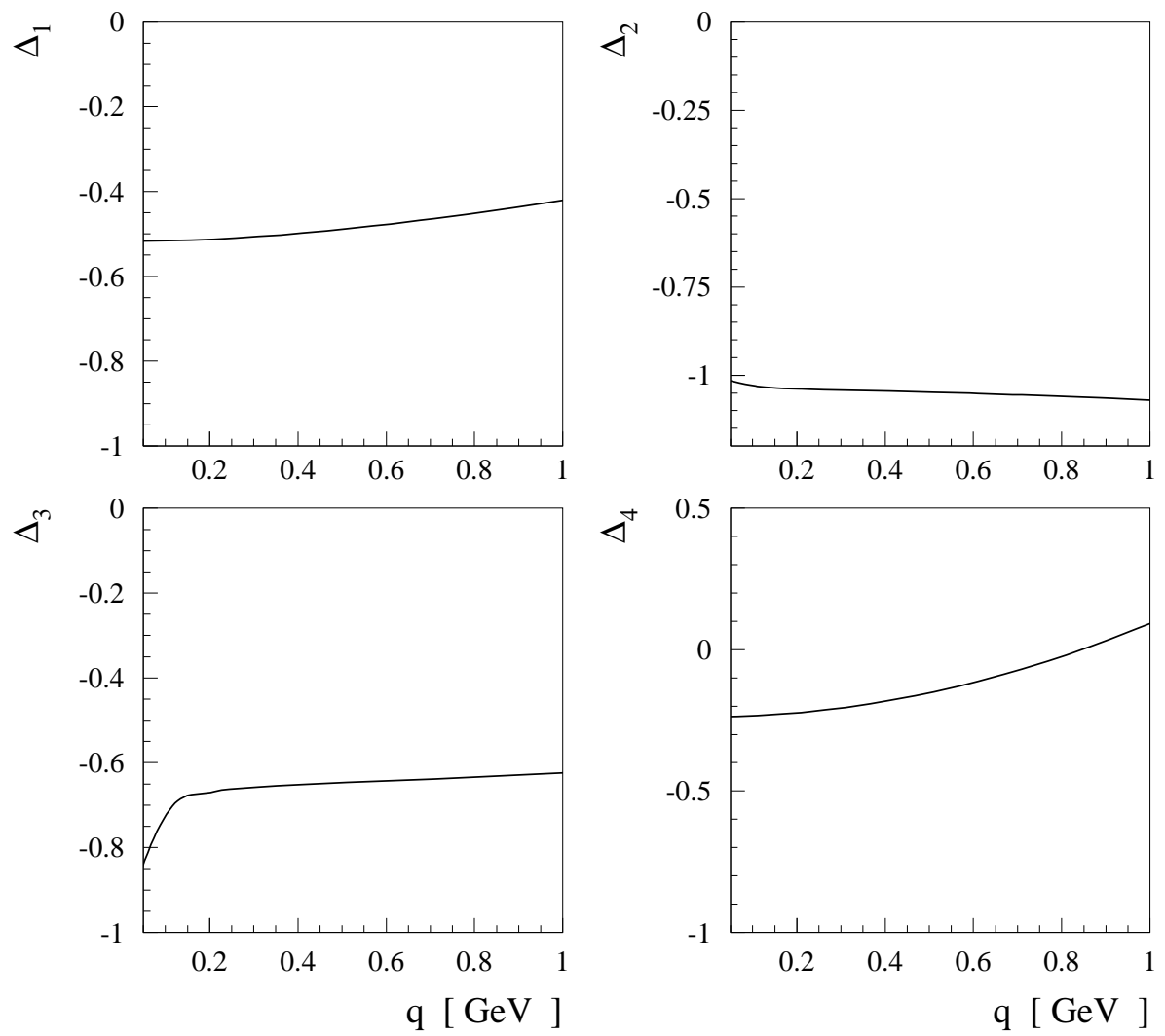

FIG. 7. Violation of the relations of Eqs. (77) - 80 anction of $\mathrm{q}=|\vec{q}|$. 